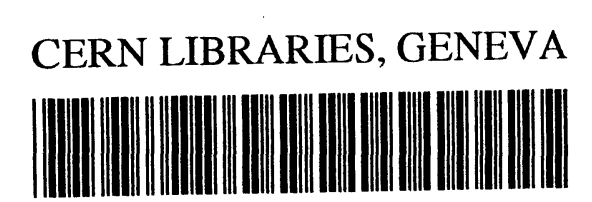

CM-P00065614

\section{HS DIVISIONAL REPORT}

17 August 1981

\title{
AN INTRODUCTION TO THE MONTE CARLO METHOD
}

\section{CERN LIBRARIES, GENEVA}

by

T. Lund, CERN, Geneva/LiTH, Linkoping

\begin{abstract}
The Monte Carlo method is a wide spread numerical method which is used in a lot of different areas of numerical calculations. This thesis gives a short introduction to the method. In the introduction we give a short resume and history of the method. One area of major interest in the use of Monte Carlo methods is the generation of random numbers and sampling from given distribution functions; this is discussed in the next chapters. Then follows a description of the method and various aspects of the performance are discussed. Variance reduction plays a big role in the Monte Carlo theory and the most common topics are covered.To illustrate the use of Monte Carlo methods we give some examples of applications from the domain of particle transport. A revision of probability theory and an mathematical justification of the method is included in the appendices.
\end{abstract}




\section{CONTENTS}

\section{Chapter}

\section{paqe}

1. INTRODUCTION . • . . . . . . . . . . . . . . . . . . . . . . 1

What is the Monte Carlo method? . . . . . . . . . . . . . 1

History . . . . . . . . . . . . . . . . . . . . 2

Further references . . . . . . . . . . . . . . . . 3

2. RANDOM NUMBERS • . . . . . . . . . . . . . . . . . . . . . 4

Random numbers . . . . . . . . . . . . . . . . . 4

Introduction . . . . . . . . . . . . . . . . . . 4

Truly random numbers . . . . . . . . . . . . . . . 5

Pseudo-random numbers . . . . . . . . . . . . . . 6

Testing pseudo-random generators . . . . . . . . . . . . 8

Quasi-random numbers . . . . . . . . . . . . . 9

Further references . . . . . . . . . . . . . . . . . 11

3. SAMPLING METHODS . . . . . . . . . . . . . . . . . . . . . 12

Introduction . . . . . . . . . . . . . . . . . . . . 12

General methods . . . . . . . . . . . . . . . . . . 12

The direct method... . . . . . . . . . . . . . . 12

The rejection method . . . . . . . . . . . . . . . 14

The composition method . . . . . . . . . . . . . 16

Ratio of uniform deviates................ . . 18

Von Neumann-Forsythe's method . . . . . . . . . . . . . . . 19

Special distributions . . . . . . . . . . . . . . . . 21

Direct method....................... . . . 21

Composition-rejection method . . . . . . . . . . . . 22

Ratio of uniform deviates . . . . . . . . . . . . . 22

Von Neumann-Forsythe's method . . . . . . . . . . . . 23

Comparison of the methods... . . . . . . . . . . 23

Fur ther references. . . . . . . . . . . . . . . . . . . 24

4. PRINCIPLES OF MONTE CARLo CALCULATIONS . . . . . . . . . . . . . . . 25

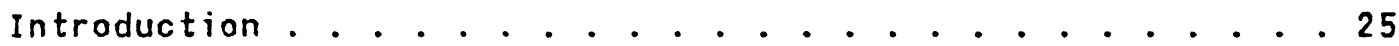

Direct simulation . . . . . . . . . . . . . . . . . . 25

Monte Carlo as numerical integration . . . . . . . . . . . 26

Examples . . . . . . . . . . . . . . . . . . . . 26 
Formulation . . . . . . . . . . . . . . 28

Numerical quadrature ................. . 31

Characteristics of the Monte Carlo method........ . 32

Further references................. 32

5. VARIANCE RedUCTION . . . . . . . . . . . . . . . . 33

Introduction . . . . . . . . . . . . . . . . 33

Stratified sampling . . . . . . . . . . . . . 33

Control variates................... 35

Importance sampling ................. 36

Antithetic variates . . . . . . . . . . . . . . 38

Regression methods . . . . . . . . . . . . . . . 39

orthonormal functions ................. 40

Conditional Monte Carlo . . . . . . . . . . . . . . 42

Further references... . . . . . . . . . . . . . 44

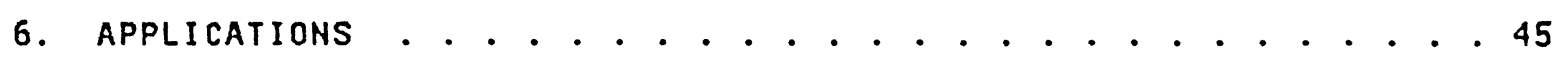

The particle transport problem . . . . . . . . . . . 45

The Boltzmann equation. . . . . . . . . . . . . . . . 46

Particle transport . . . . . . . . . . . . . . . 47

Geometry . . . . . . . . . . . . . . . . 44 4

Time . . . . . . . . . . . . . . . . . . . . 4 47

Next interaction ................. 48

Interaction.................... . 49

Estimators ................... 50

Variance reduction................... 51

Splitting and Russian roulette ............ 51

The exponential transform . . . . . . . . . . . . 52

Particle cutoffs .................. 52

Software....................... 53

Fur ther references................. 53

Appendix

paqe

A. PROBABILITY THEORY . . . . . . . . . . . . . . . 54

Properties of random variables.............. . 54

Common distributions.................. 56

Limit theorems . . . . . . . . . . . . . . . . 57

B. Mathematical basis . . . . . . . . . . . . . . . . . 58

Mathematical properties ................ 58

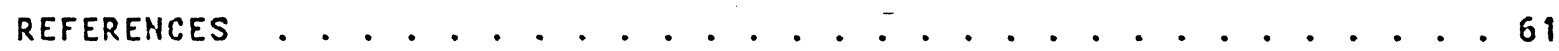


Chapter 1

INTRODUCTION

\subsection{WHAT IS THE MONTE CARLO METHOD?}

The Monte Carlo method is a method of approximately solving problems in mathematics and physics by simulating the problem by using random numbers. Or, stated in a more probabilistic way by Halton [24]:

The Monte carlo method is defined as representing the solution of a problem as a parameter of a hypothetical population, and using a random sequence of numbers to construct a sample of the population, from which statistical estimates of the parameter can be obtained.

The Monte Carlo method has been used as a branch of numerical analysis in many areas e.g. nuclear physics and numerical integration where the resources of theoretical mathematics are not immediately available. As one could believe the Monte Carlo method is not appropriate only to solve problems that have a probabilistic nature. The applications rather depend on our ability to formulate. the problem in such a way that we can obtain the solution by playing games with random numbers. In fact sometimes it is better (in some sense) to reformulate the probabilistic problem into a more artificial model. Thus the Monte carlo method can be seen as a universal method for solving mathematical problems.

The method consists in short of setting up a stochastic model and then by sampling from appropriate probability distributions we estimate the required numerical solution by statistical means. The next two chapters deals with the generation of random numbers and the sampling from distribution functions. In chapter 4 we will discuss the formulation of the method and in the next chapter various aspects of convergence acceleration (in the literature usually called variance reduction) will be examined. In the last chapter we discuss an application of the Monte Carlo method from the fields of particle transport. A short overview of probability theory and a discussion of the mathematical justification of the method could be found in the appendices. 


\subsection{HISTORY}

The Monte Carlo method has been traced as far back as Babylonian and 0ld Testament times. During the eighteenth and nineteenth centuries one tried to calculate the value of $\pi$ by using a procedure known as "Buffons needle"[6]. The calculation was performed by throwing a needle in a random way onto a board ruled with parallel lines and counting how many times the needle intersected the lines.

In the beginning of our century statistical problems were solved with the help of random numbers. Their work was mainly concerned with testing theoretical results. In 1908 student [48] discovered the distribution of the correlation coefficient by using experimental sampling. He started with an initial guess based on the knowledge of different moments of how it would look like and then fitted samples of different sizes to $h$ is model. Then he approximated the estimates of the parameters and made a guess of their values. This guess happened to be the exact theoretical result. This is an example of an unusual way to use the Monte Carlo method since nowadays there is little attempt to guess the exact result. This use of the Monte Carlo method had one drawback: It was hard to improve the procedure to get more accurate results without guessing.

However, the more systematic use and development dates from the end of the second world war. It was with the design of the atomic bomb that the first practical problems were treated with the Monte Carlo method. This work involved direct simulation of the probabilistic problems concerned with random neutron diffusion in fissile material. These simulations, performed by von Neumann and Ulam, also included some variance reducing techniques such as "Russian roulette" and "splitting". However, the reduction techniques were first investigated by Harris and Kahn in 1948. In the 1940s some work on deterministic problems were also made, e.g. Fermi use the Monte Carlo method to obtain estimates of the eigenvalues of the Schrodinger equation.

During the 1950s the Monte Carlo method was intensively studied in the USA. However, these efforts did not always serve the Monte Carlo method. In fact there was an attempt to solve every problem in sight with Monte carlo without paying enough attention on which problems it could solve efficiently. Numerical analysts were not late to point out those problems where the Monte Carlo method was inferior to already existing numerical methods. But, by a better recognition of those problems in which the Monte Carlo method is a good method, and sometimes the only, it has nowadays a some what better reputation.

The development of the Monte Carlo method has been closely related to the apparition of fast computers since the simulation of random quantities by hand is a very laborious process. The computers has been used both in judging the usefulness of the techniques and in developing the theory. The study of the Monte Carlo method is one good example of the creative use of computers as a research tool. In our days the Monte Carlo method draws on an extremely broad range of mathematical disciplines, from probability theory to number theory and from mathematical analysis to numerical analysis. 
Like numerical analysis in general, the study of the Monte Carlo method has produced a great number of specialized techniques. And it is only recently that certain underlying structures and regularities have began to emerge. Some guidelines for the future study of the Monte Carlo method could be stated. First there is the study of the Monte Carlo method as a branch of probability and the quasi-Monte carlo method as a branch of numerical analysis and especially multidimensional quadrature. Secondly the studies could be split into Monte Carlo processes and random generators. The future will show if the deterministic numerical techniques will become so powerful that they will be able to handle any problem to which the Monte Carlo method is suited or if the method will survive.

\subsection{FURTHER REFERENCES}

A general treatment of the Monte Carlo method, including overviews of the history, can be found on [26], [43] and [44]. The original paper that gave the method its name is written by Metropolis and Ulam [34]. The history of the method and the problems that were discussed during the 50 s could be found in [35]. Halton[24] gives a long list of questions that could be subjected to further research. 
chapter 2

RANDOM NUMBERS

\subsection{RANDOM NUMBERS}

\subsubsection{Introduction}

In all Monte Carlo calculations we have the common feature that at some point in the procedure we have to substitute for a random variable a set of actual values, distributed according to this random variable. These actual values could have been produced by some random process and because of that we call them random numbers. However, the numbers are not generally produced in this way but rather by some algorithm appropriate for generating numbers on a computer. These numbers are submitted to various statistical tests to assure that they have the properties that we require. But since we are dealing with computers we are restricted to only finitely many of these numbers (therefore we call them pseudo-random numbers) and to apply statistical tests to deterministic sequences to get some probabilistic "level of significance" could then not give us more than a qualitative justification. As an ultimate test on the random numbers we have the Monte Carlo problem itself. If it gives a reliable answer to the problem all other tests are unnecessary since it was just this final solution that we wanted.However, this poses other problems: How reliable is the answer? Can we get an estimation of the error? The usual way to solve this is to assume the randomness of the numbers and the apply various statistical theorems to get an error estimate. Another way is to reject the use of pseudo-random numbers and instead using quasi- random numbers with known properties e.g. equidistribution. Then we can obtain error estimates by using concepts as discrepancy, variation etc. (see Appendix $B$ ). This latter approach is well treated in [50]

Thus, we are dealing with three different types of sequences: Truly random, pseudo-random and quasi-random sequences. In the following part of this chapter we will discuss each of them. The distribution function we are most interested in when dealing with random numbers is the uniform distribution which is defined as

$$
F(y)=\begin{array}{ll}
0 & y<0 \\
y & 0 \leq y \leq 1 \\
1 & y>1
\end{array}
$$

and we will denote a random number drawn from this distribution by $\xi$. 


\section{1 .2 Truly random numbers}

A sequence of truly random numbers is unpredictable and therefore unreproducible. Such a sequence can only be generated by some physical process e.g. radioactive decay or thermal noise in electronic devices. If such a physical process is used to generate the random numbers for a Monte Carlo calculation there is no theoretical problem with our statistical justifications.

The earliest generators were typically based on gambling devices where the randomness were introduced by a human operator. These were e.g. dices (cubic or icosahedral), coins and the Monte Carlo roulette wheel. Another source of truly random numbers are the different $k$ inds of tables of random numbers. These are either compiled from different random sources (such as census reports) or obtained from some specially constructed physical devices.

The normal way to use a physical device is the following:

Count the impulses produced by a random noise source e.g. cosmic ray arrival times or thermal noise, in fixed time intervals. If after each time interval, the counted number is odd then produce a "l" otherwise produce a "0".

The properties of the counter and of the random source usually introduce a bias. But this can easily be removed. If we, for instance, produce a "l" with probability $p$ and a " 0 " with probability $q=1-p$, and we assume that the digits are independent, then we form a new sequence as follows:

For each generated pair of digits we have

$\begin{array}{lll}1 & 1 & \text { with probability } \\ 1 & 0 & \text { with probability } \\ 0 & 1 & \text { with probability } \\ 0 & 0 & \text { with probability } \\ q^{2}\end{array}$

If the digits in a pair is the same then reject them. If we got a "I 0 " then produce a " 1 " and produce a "0" if we got the pair "0 1". Then, clearly, we get "0"s and "l"s with the same probability. The efficiency" of this procedure could be quite low (always $\leq 25 \%$ ) but we avoid the problem of knowing, in advance, the time dependence of the bias.

In practice, however, truly random number generators are seldom used mainly due to three difficulties: It is difficult to construct physical devices which are stable and do not demand too frequent testing, the devices are too slow (so $f a r$ ) to be used in connection with a computer and they are non-reproducible. ${ }^{2}$ However, the doubts on the pseudo-random

1 The efficiency is the ratio of the total number of produced digits to the total number of supplied digits. 
number generators, due to the more exact theoretical discussion of them, might lead to a more intense research for truly random generators.

\subsubsection{Pseudo-random numbers}

The most commonly source of random numbers are those called pseudo- random numbers. These are generated according to a mathematical formula and are therefore determined "in advance" and thus not at all random. But they are supposed to be indistinguishable from a sequence generated truly randomly. The pseudo-random number generators could be thought of as a general purpose device whose output "looks random". Originally these generators were subjected to and expected to pass the same statistical tests as true random numbers. Nowadays, somewhat different criteria are also applied (see below).

The two main types of arithmetic procedures that have been used are the middle-square method and linear recurrence relations. The former is most of historical interest whereas the latter is the most examined and successful method.

The middle-square method, proposed by von Neumann, appears to be the first method used to produce pseudo-random numbers. Assume that all numbers have $2 t$ digits and are represented in the base $R$. To obtain the next random numbers, $x_{n+1}$, from $x_{n}$ we square $x_{n}$ to get a product with $4 t$ digits (eventually by left adjusting with $0^{\prime} s$ ). Then we remove the $t$ most significant and the $t$ least significant digits and letting the middle $2 t$ digits be our random number $x_{n+1}$. This may be expressed by the formula

$$
x_{n+1}=\left[x^{2} n^{\prime} / R^{t}\right]-\left[x^{2} n^{\prime} R^{3 t}\right] \cdot R^{2 t}
$$

where $[x]$ denotes the integer part of $x$.

By a judicious choice of starting number one can get a rather long string of numbers which appear random but the generator is not a good one and it is not used any longer. It happens that degeneracy to zero or some other value (e.g. 50 in the case $R=10, t=1)$ occurs in ways that are difficult to predict. Some variations of $(2.2)$ has been proposed but no great improvements have been found.

In 1951, Lehmer[32] suggested a method known as the multiplicative congruential or the linear congruential method. It is defined by

$$
x_{n+1} \equiv \lambda x_{n}(\bmod M)
$$

where $a \equiv b(\bmod m)$ signifies that the rest when $a-b$ is divided by $m$ is 0 . This has subsequently been generalized to the mixed-congruential method

2 The reproducibility is desired for test reasons. 


$$
x_{n+1} \equiv \lambda x_{n}+\mu(\bmod M)
$$

and to the even more general form of a linear recurrence relation

$$
x_{n+k} \equiv \lambda_{0} x_{n}+\lambda_{1} x_{n+1}+\ldots+\lambda_{k-1} x_{n+k-1}+\mu(\bmod M)
$$

The generators (2.3)-(2.5) have been extensively examined ( see e.g. (281) and the best choices for $\lambda$ and $\mu$ in (2.3) and (2.4) has been assured by a mixture of empiricism and number theory. The class of generators (2.5) has not been as widely used as the other two because they do not seem to give improvements proportional to their greater complexity.

The criterion for the best choices of $\lambda$ and $\mu$ has been that the recurrence relation should generate a sequence, necessarily periodic with period $\leq M$, with as large period as possible. We give the results (2.3) in the most interesting cases and for (2.4) in general.

1. The maximum period of the sequence generated by (2.3) is attained under the following conditions:

i) If $M=2^{t}$ and $t \geq 3$ then $\lambda$ yields the maximum period of $2^{t-2}$ if and only if $\lambda \equiv \pm 3(\bmod 8)$.

ii) If $M=10^{t}$ and $t \geq 4$ the maximum period $5 \cdot 10^{t-2}$ is attained if $\lambda \equiv \pm 3, \pm 13, \pm 27, \pm 37, \pm 53, \pm 67, \pm 77, \pm 83(\bmod 200)$

2. The maximum period of the mixed-congruential generator (2.4) is $M$ if $\mu \neq 0$ and

i) $\mu$ is relatively prime to $M$.

ii) $\mu \equiv 1(\bmod p)$ for every prime factor $p$ of $M$.

iii) $\mu \equiv 1(\bmod 4)$ if $M \equiv 0(\bmod 4)$

These choices of $\lambda$ and $\mu$, giving the maximum period, ensure that the numbers produced are uniformly distributed. However, they may nevertheless be highly correlated and the correlations should be examined. One method to investigate the maximum period generators is to submit them to "tests for randomness". But we could also make theoretical investigations of the correlation and of the autocorrelation function. This would give us the possibility to find the good and bad multiplicators. This work has been done by Dieter and Ahrens $[3,11,12]$ for the multiplicative generator (2.3). They have found that the best simple multiplicative generator is that with the multiplier

$$
\mu=2^{t-2} \cdot(\sqrt{5}-1) / 2
$$

where $t$ is the integer bit length in the computer at hand. 
In practice the constant $\mu$ is determined for a given value of $t$ by multiplying $2^{t-2}$ into a very precise value of the constant $(\sqrt{5}-1) / 2$ (the golden section ratio) and then rounding to the nearest integer congruent to 5 (mod 8 ). We then get the maximal period of $2^{t-2}$.

\subsubsection{Testing pseudo-random qenerators}

In the early days one had no deeper comprehension of the behaviour of the pseudo-random number generators. Thus, it was necessary to let the generators pass some "tests of randomness" in order to get a measure of the "goodness". These tests consisted of forming some function of the generated sequence and then comparing the result with the known expectation of the same function applied to true random numbers. Various types of tests have been created, some tests seeming to be more sensitive than others, but since the behavior of "non-randomness" is not known in advance it is not possible to measure the power of any test in a precise way. A description of some tests may be found in [28] and Knuthl30] gives a good summary of tests and techniques.

Among the various tests we may mention frequency tests (proportion of occurences of digit values), serial tests (proportion of occurences of pairs of digits), poker tests (occurences of poker like blocks of digits), gap tests (e.g. length of gaps between successive zeros) and run tests (length of sequences with decreasing or increasing digits or block of digits). One test which is "good" in the sense that we get a little bit more insight into the properties of the generator and which is rather sensitive to the kind of "non-randomness" that we are interested in is the visual test. Take pairs of numbers, chosen in an appropriate way, as coordinates in a plane and plot these points onto a visual display. Then we can study e.g. the uniformness of the sequence and the correlation between generated points.

A number of more rigorous approaches, not based on statistical tests has been made. We will look at one of these, the uniform distribution modulo one. Let $\Xi=\{\xi i\}, i=1,2, \ldots$ be an infinite sequence of numbers and let any subsequence of $k$ numbers $\left\{\xi_{n+1}, \xi_{n+2}, \ldots, \xi_{n+k}\right\}$ have the probability $a_{1} a_{2} \cdots a_{k}$ of lying in the "hyperbrick"

$$
0 \leq \xi_{n+i}<\alpha_{i} \leq 1 \quad i=1,2, \ldots, k
$$

Then we say that any sequence $\Xi$ of points in $I=[0,1]$ is k-equidistributed if for every choice of $a_{1}, \alpha_{2}, \ldots, a_{k}$ in $[0,1]$

$$
\lim _{m \rightarrow \infty}\left(v_{m}\left(E\left(a_{1}, a_{2}, \ldots, a_{k}\right)\right) / m\right)=a_{1} a_{2} \cdots a_{k}
$$

where $E\left(a_{1}, a_{2}, \ldots, a_{k}\right)$ is the event in (2.7) and $y_{m}$ is the number of points among $\xi_{1}, \xi_{2}, \ldots, \xi_{m}$ which fall in the region corresponding to $E$. 
We also say that $\Xi$ is completely equidistributed if it is k-equidistributed for every integer $k \geq 0$. In the discrete case we can define equidistribution as follows: Let $A=\{0,1 / M, 2 / M, \ldots,(M-1) / M\}(e . g$. the points generated by a psuedo-random number generator). Then there exist $M^{p+1}$ different $(p+1)$-tuples $(p=0,1, \ldots)$ with elements from $A$. If each of these $(p+1)$-tuples occurs with the same frequency in an unlimited sequence of generated numbers, this sequence is said to be $(p+1)$-equidistributed

A number of results has been obtained on the equidistribution properties of various pseudo-random number generators. For the generator (2.3) we know that it is not k-equidistributed for any $k>1$ and that it may fail to be 1 -equidistributed even if $x_{0}$ is transcendental. One sequence which is completely equidistributed when $\theta$ is transcendental is

$$
x_{n}=\left\{\theta^{n}\right\}
$$

where $\{x\}$ denotes the fractional part of $x$. However, there is none explicit $\theta$ know for which (2.9) is completely equidistributed sequence but such a sequence can nevertheless be constructed ( see (31)) even though the algorithm could not be used for any practical calculations. Another 1-equidistributed sequence is the Weyl sequence, which was discovered by Weyl, the originator of the work on equidistribution, (see $[49]$ ). It is defined as

$$
x_{n}=\{n a\}
$$

where $\alpha$ is irrational.

These results on equidistributed sequences seem difficult to apply since the theory only covers the asymptotic properties of infinite sequences. The most important result is that reasonably complete and accurate a priori predictions of the performance of various generators can be made.

\subsubsection{Quasi-random numbers}

The various deficiencies of pseudo-random number generators discussed above have given $r$ ise to another type of sequence known as quasi-random numbers. These sequences do not pretend to have any appearance of randomness but they rather assure that they have the property of producing the desired result. In a way they are then more satisfactory than the pseudo-random numbers since they are not based on an illusion but on the other hand the generation of the sequences are more dependent on the problem at hand. The quasi-random sequences are related to the behavior of the error

$$
\begin{aligned}
& \delta_{m}(f)=\mid \sum_{m=1}^{1} \sum_{n=1}^{m} f\left(\xi_{n 1}, \xi_{n 2}, \ldots, \xi_{n k}\right)- \\
& -\int^{\prime} d z_{1} \quad 0 f^{\prime} d z_{2} \cdots 0_{0} f^{\prime} d z_{k} f\left(z_{1}, z_{2}, \ldots, z_{k}\right) \mid \\
& \text { - } 9 \text { - }
\end{aligned}
$$


For a given class of functions, $f$, we examine $\delta_{m}(f)$ for different choices of sequences $\Xi=\left\{\xi_{i}\right\}$ and the smaller $\delta_{m}(f)$ the better is the sequence $\Xi$ for integrating functions of the given class. The problem is then to find a sequence that minimizes $\delta_{m}(f)$. Before we give some results on the sequences $\Xi$ we introduce the notion of local discrepancy and mean-square discrepancy. Let $\underline{\alpha}=\left[\alpha_{i}\right], i=1, \ldots, k$ and let $V(\underline{a})=\mathbb{I}$ $a_{i}$ be the volume of the region in (2.7). If we group the $\xi i$ 's in the sequence $\Xi$ into $k$-tuples $\xi_{n}=\left[\xi_{n i}\right], i=1, \ldots, k$, the sequence $\Xi$ becomes a sequence of points in the hyperunit cube $\left\{x_{i}, i=1,2, \ldots, k \mid 0 \leq x_{i} \leq 1\right\}$. Then we define, with $\nu_{m}(E)$ as given above, the local discrepancy as

$$
\Delta_{m}(\underline{a})=y_{m}(\underline{a}) / m-V(\underline{a})
$$

The mean-square discrepancy, $J$, is then given by

$$
J_{m}^{2}=\text { of } \int^{1} d a_{1} \text { of } f^{1} d a_{2} \cdots o f^{1} d a_{k} \mid \Delta_{m}(\underline{a})^{2}
$$

Sequences with satisfactory low discrepancies can be found and these sequences can be used for integrating functions, which are sufficiently smooth, in the hypercube. A lower bound on $J_{m}$ for every sequence $\Xi$ whatever has been given by Rothl41] as

$$
J_{m}>c_{k}(\ln m / m)(k-1) / 2
$$

where $c_{k}$ is a constant.

An example of a sequence with low discrepancy is the van der corput's sequence. Suppose that the natural numbers, $n$, are expressed in the base $r$ as

$$
n=n_{0}+n_{1} r+n_{2} r^{2}+\ldots+n_{m} r^{m}
$$

where $n_{m} \neq 0$ and $m=\left\{\log _{r} n\right\}$

Next we form a number in $(0,1)$ by writing the digits $n_{i}$ in reverse order, preceded by a point. This gives the numbers

$$
\phi_{r}(n)=n_{0} r^{-1}+n_{1} r^{-2}+\ldots+n_{m} r^{-m-1}
$$

Then the van der corput sequence is the sequence $(r=2)$

$$
\equiv=\left[p_{2}(n)\right]_{n=0}^{2 m-1}
$$

This can then be extended to $k$ dimensions giving the sequence of vectors

$$
\left(\phi_{r_{1}}(n), \phi_{r_{2}}(n), \ldots, \phi_{r k}(n)\right) \quad n=0,1, \ldots
$$

where the ri are all pairwise relatively prime. 
If we calculate the mean-square discrepancy $J_{m}$ for this sequence we arrive at the result

$$
J_{m}<c_{k}(\ln m)^{k / m}
$$

where $C_{k}$ is a constant.

This should be compared with the expected value of $\mathrm{J}_{\mathrm{m}}{ }^{2}$ in the case where $\Xi$ is a true random sequence. This value is the much greater quantity

$$
E\left[J_{m}^{2}\right]=\left(2^{-k}-3^{-k}\right) / m
$$

\subsection{FURTHER REFERENCES}

There are a lot of papers written on the behaviour of pseudo-random sequences. A classical paper is the one written by Marsaglial33]. In [51] there are articles which treat both pseudo-random and quasi-random sequences. Franklinl19] discusses the properties of random sequences and Halton[25] gives an algorithm for producing a van der corput sequence. 
SAMPLING METHODS

\subsection{INTRODUCTION}

We have so far just been dealing with the generation of a uniformly distributed random variable. In many applications, however, random numbers with other distributions are of interest and the question is then how to generate random numbers distributed according to a general density function. This is done by taking uniformly distributed numbers and then, in one way or another, transform them to numbers $w i$ th the desired distribution. In the following we first describe some general methods of performing the transformation and then we discuss the sampling from some special distributions. We write $\xi$ for a uniformly distributed random number and let $n$ denote a random number with density function $f(y)$ and distribution function $F(y)$.

\subsection{GENERAL METHODS}

\subsubsection{The direct method}

Suppose we have $n$ events $x_{1}, x_{2}, \ldots, x_{n}, e a c h$ of them occuring with the probabilities $p_{1}, p_{2}, \ldots, p_{n}$ and with $\Sigma p_{i}=1$. If we want to choose an event randomly we choose a random number, $\xi, 0 \leq \xi<1$. Then we clearly have that the relation

$$
\sum_{n=1}^{i-1} p_{n} \leq \xi<\sum_{n=1}^{i} p_{n}
$$

determines the event $x_{i}$ so that $n=x_{i}$.

If we on the other hand have a continuous variable from which we want to sample then we could approximate the density function with a piecewise constant function and then just replace the sums in (3.1) by integrals. We then get the equation

$$
\xi=\int_{x_{0}}^{x} f(y) d y=f(x)
$$

If the distribution function $F$ has a know inverse $F^{-1}$ we get our random number as 


$$
n=F^{-1}(\xi)
$$

The usefulness of this relation highly depend on the function $F^{-1}$. And in practice it is rare that one finds the inverse. But we can still use the relation ( 3.2 ) if we are able to calculate $F(x)$ for different values of $x$ (e.g. direct or by Gaussian quadrature). We divide the interval $[0,1]$ into subintervals and for each endpoint of these intervals we determine $x$ as the solution of (3.2) (e.g. with Newton-Raphson's method). Then we can sample $\xi$ and get $n$ as the interpolated $x$-value. With a clever subdivision of the interval $[0,1]$ this method could be very fast. What we do is an approximation of the distribution function by a piecewise linear function. If this does not give enough accurate values we could make the approximation with functions of higher degrees e.g. splines.

An often encountered problem is to sample a uniform random variable which is uniform on the interval $[a, b]$ instead of on $[0,1]$. This is an example where we can use the direct method. The density function is now

$$
f(y)=1 /(b-a) \text { for } a \leq y \leq b
$$

and if we integrate from a to $y$ we get the distribution function as

$$
F(y)=(y-a) /(b-a)
$$

We then calculate the inverse and as the result we get our sample from the uniform distribution on $[a, b]$ as

$$
n=a+(b-a) \xi
$$

As another example of the direct method we show how to sample from the exponential distribution, with density function

$$
f(y)=\lambda \operatorname{Exp}(-\lambda y) \text { for } y<0
$$

where $\lambda$ is a parameter.

We get the distribution function as

$$
F(y)={ }_{0} \int y+(t) d t=1-\operatorname{Exp}(-\lambda y)
$$

and the inverse is

$$
F^{-1}(y)=-\ln (1-y) / \lambda
$$

Now, since $\xi$ is distributed as $1-\xi$, we get the following sampling scheme

$$
n=-\ln (\xi) / \lambda
$$




\subsubsection{The rejection method}

A method often convenient for sampling from a density function $f(y), a \leq y \leq b$, when $f$ is an easily computable function is the rejection method. Suppose that $f(y)$ is bounded i.e. $0 \leq f(y) \leq c<\infty$ and that it is zero outside the interva] $[a, b]$. The method is then (see fig. 1)

1. Generate a pair of random numbers $\xi_{1}, \xi_{2}$

2. Calculate $y=a+(b-a) \xi 1$, i.e. $y$ is uniformly distributed in $[a, b]$

3. Calculate $z=c \xi_{2}$, i.e. $z$ is uniformly distributed in $[0, c]$

4. If $z>f(y)$ then reject the pair and goto step 1

5. Accept $n=y$ as the wanted random number.

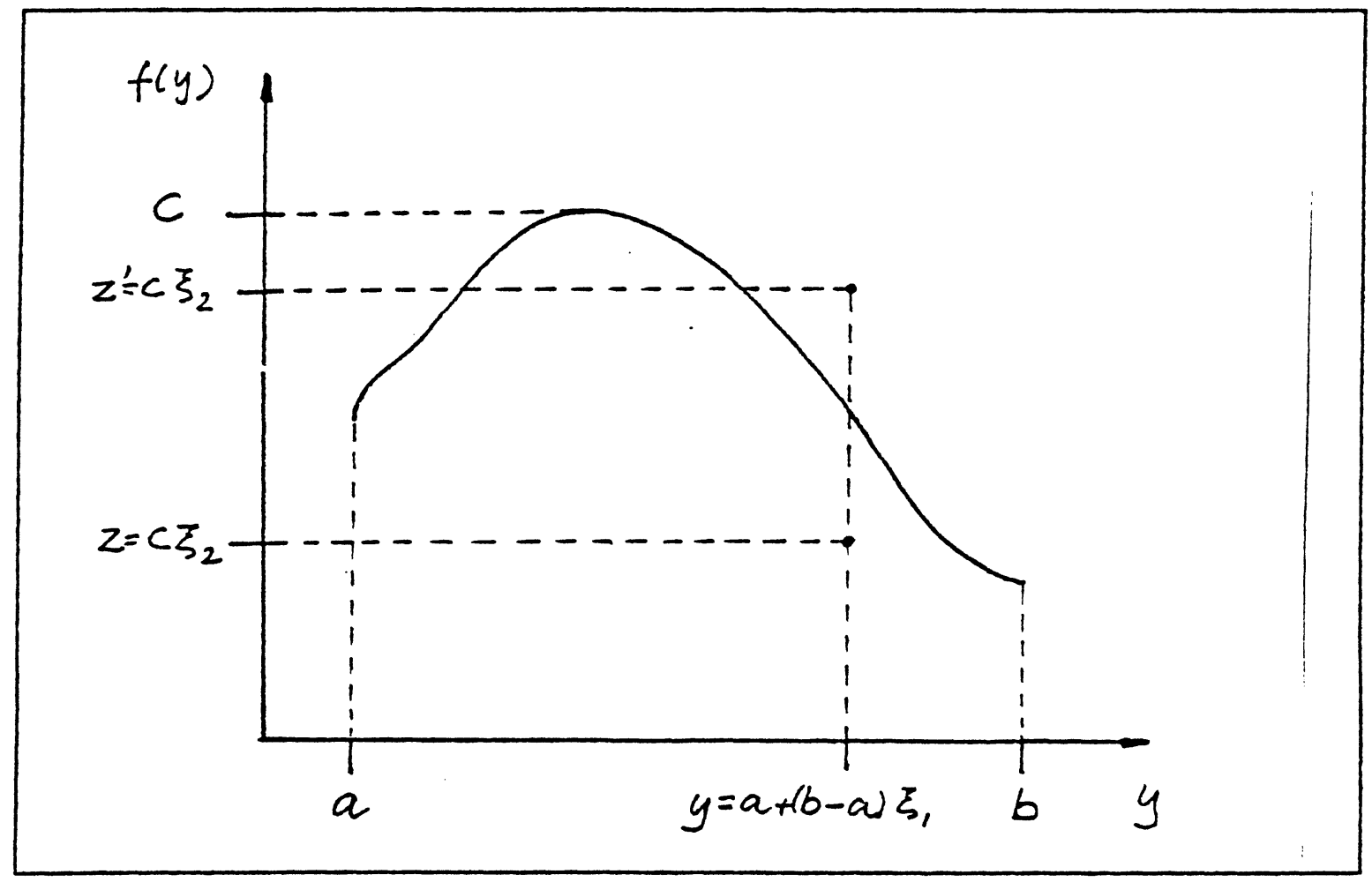

Figure 1: The rejection method

The efficiency of the rejection method is defined as the ratio of the number of accepted values $n$ to the total number generated $n^{\prime} s$. And this is clearly the ratio of the area under the curve in fig.l to the total area of the rectangle $[a, b] \times[0, c]$ i.e. 


$$
\text { Efficiency }={ }_{a} \int^{b} f(y) d y / c(b-a)=1 / c(b-a)
$$

The efficiency, which is obviously less than 1, indicates to what extent a given rejection method may be feasible. If the efficiency is to low too much time will be spent on rejections and this is unacceptable. Many commonly used rejection methods have an efficiency considerably greater than $50 \%$.

If we want to use the rejection method but the efficiency is too low then we can get around this problem by a simple trick. Call the region below the function $\Omega$ i.e. $\Omega=\{y: 0 \leq y \leq f(y)\}$. We then divide $\Omega$ into disjoint regions $\Omega_{i} i . e$.

$$
\Omega=\bigcup_{i=1}^{n} \Omega_{i} \text { and } \Omega_{i} \cap \Omega_{j}=\phi \text { if } i \neq j
$$

Then we enclose each $\Omega_{i}$ in a region $R_{i}$ and calculate $n$ in the following manner:

1. Select region $R_{i}$ with probability $p_{i}=\left|\Omega_{i}\right| /|\Omega|$

2. Use the rejection method above in the region $R_{i}$.

Since we have diminished the total area of the rectangle $[a, b] \times[0, c]$ by this subdivision the ratio of the areas will be greater and we have augmented the efficiency.

In the rejection step (step 4 above) we have to evaluate the function $f$. In some cases this could be a very expensive operation. We could then include some simple pre-tests so that we only have to evaluate $f$ a comparatively small number of times. These pre-tests could for example be some easily computable bounds, $f_{1}$ and $f_{2}$, on $f$ such that

$$
f_{1}(y) \leq f(y) \leq f_{2}(y)
$$

Then we only need to make the costly evaluation of $f$ if we have passed the pre-tests i.e. if $f_{1}(y)<z<f_{2}(y)$ ( $z$ as in step 4 above).

Consider the problem of the evaluation of $\cos \theta$ and $\sin \theta$ for $a$ uniformly distributed angle $\theta$. We generate $\xi_{1}$ and $\xi_{2}$ and thus determining $a$ point in the unit square. If $\xi_{1}{ }^{2}+\xi_{2}{ }^{2}$, 1 then we reject the point and repeat the test with a new point otherwise the point is inside the unit circle and we take

$$
\begin{aligned}
& \cos (\theta / 2)=\xi_{1} / \sqrt{\left(\xi_{1}^{2}+\xi_{2}^{2}\right)} \\
& \sin (\theta / 2)=\xi_{2} / \sqrt{\left(\xi_{1}^{2}+\xi_{2}^{2}\right)}
\end{aligned}
$$

Using trigonometric formulas we get $\theta$ in the interval $[0, \pi]$ and the more efficient calculable formulas

$$
\begin{aligned}
& \cos \theta=\left(\xi_{1}^{2}-\xi_{2}^{2}\right) /\left(\xi_{1}^{2}+\xi_{2}^{2}\right) \\
& \sin \theta=\xi_{1} \xi_{2} /\left(\xi_{1}{ }^{2}+\xi_{2}{ }^{2}\right)
\end{aligned}
$$


To get $\theta$-values in the interval $[0,2 \pi]$ we add a randomly chosen sign on $\sin \theta$. The efficiency of this method is the ratio of the area of a circle with radius 1 to that of a square with side 2 so we get it to $\pi / 4 \simeq 79 \%$ which is an acceptable value.

\subsubsection{The composition method}

This method is applicable when $f(y)$ can be written in the form

$$
f(y)=\int g_{z}(y) d H(z)
$$

where $\left\{g_{z}(y)\right\}$ is a family of density functions and $H(z)$ is a distribution function from all of which we know how to sample. The procedure is then to sample $z$ from $H$ and then to sample the desired $n$ from the corresponding distribution $g z$.

As an example of this method we look at the case when $f(y)$ is restricted to a range where it has a power series expansion i.e.

$$
f(y)=\sum_{n=0}^{\infty} a_{n} y^{n}
$$

where $0 \leq y<1$ and $a_{n} \geq 0 \forall n$

In this case we may choose $n$ from a discrete distribution with frequency function

$$
p_{n}=a_{n} /(n+1) \cdot s
$$

where $S=\Sigma_{i} a_{i} /(i+1)$

and then sample $n$ from the distribution

$$
g_{n}(y)=(n+1) y^{n}
$$

which may be performed by taking $n=\max \left(\xi_{1}, \xi_{2}, \ldots, \xi_{n+1}\right)$

In this way we can sample from the density function

$$
f(y)=e^{y /(e-1)}=(e-1)-1\left(1+y+y^{2} / 2 !+\ldots\right)
$$

where $0 \leq y<1$.

and by performing a second composition, now with

$$
g_{m}(y)=\left(e^{m-y}\right) /(e-1) \quad m-1 \leq y<m
$$

and

$$
p_{m}=(e-1)^{-m} \quad m=1,2, \ldots
$$


we get the exponential density function as

$$
f(y)=\Sigma g_{m}(y) p_{m}=e^{-y} \quad 0 \leq y<\infty
$$

Thus, an exponential number, $n$ can be obtained as

$$
n=m-\max \left(\xi_{1}, \ldots, \xi_{n}\right)
$$

where $m$ and $n$ are distributed with frequencies

$$
p_{m}=(e-1) e^{-m}
$$

and

$$
q_{n}=1 /(e-1) n !
$$

respectively.

The composition method and the rejection method can be combined in many ways depending on the problem at hand. One of these combinations is the following procedure.

Suppose that $f(y)$ can be written as

$$
f(y)=\sum_{k=1}^{n} a_{k} f_{k}(y) g_{k}(y)
$$

where $a_{1}, \ldots, a_{n}$ are positive constants, $\left\{f_{k}(y)\right\}$ are density functions from which we know how to sample and $\left\{g_{k}(y)\right.$ \} are functions taking values in the interval $[0,1]$. Then we obtain our sample value $n$ with the algorithm below.

1. Select $\xi_{1}$ and $\xi_{2}$.

2. [Determine $k$ ] With $p_{i}=a_{i} /\left(a_{1}+\ldots+a_{n}\right) k=i$ is given by the relation $p_{1}+p_{2}+\ldots+p_{i-1} \leq \xi_{1}<p_{1}+\ldots+p_{i}$

3. [Sample from $f_{k}$ ] Generate $n$ from $f_{k}(y)$.

4. Calculate $g_{k}(n)$.

5. [Test] If $\xi_{2}>g_{k}(n)$ reject $\xi_{1}$ and $\xi_{2}$ and goto step 1 .

6. [Accept] Accept $\eta$ as the wanted random number. 


\subsubsection{Ratio of uniform deviates}

This method could be seen as a variation of the rejection method. It consists of replacing the problem of sampling from the distribution function $F$ by the simpler problem of uniform sampling from a corresponding irregular region, $\Omega$, in the plane. A deviate with the required distribution is given by the ratio of the coordinates of a random point in $\Omega$. The uniform sampling from $\Omega$ is often best performed with some rejection technique.

We want to generate a random variable $x$ with the density function $f(x)$ and we define the region $\Omega$ as

$$
\Omega=\{(u, v): 0 \leq u \leq \sqrt{f(v / u)}\}
$$

Then if $(U, V)$ is a pair of random variables uniformly distributed over $\Omega$ the random variable $x=V / U$ has the required density function $f(x)$. To see this we show that if $f(x)$ is a non-negative function with

$$
\int f(x) d x=k<\infty
$$

then $x=V / U$ has the density function $g(x)=K^{-1} f(x)$

The joint distribution of $(U, V)$ is

$$
\begin{aligned}
g_{1}(u, v) & =u|\Omega| & & \text { if }(u, v) \in \Omega \\
& =0 & & \text { otherwise }
\end{aligned}
$$

where $|\Omega|$ is the area of $\Omega$

If we change variables in (3.30) with the transformation $u=y$ and $v=x y$ we get the joint density function for $(X, Y)$ as

$$
\begin{aligned}
g_{2}(x, y) & =y /|\Omega| & & \text { if } 0 \leq y \leq \sqrt{f(x)} \\
& =0 & & \text { otherwise }
\end{aligned}
$$

The marginal density, $g(x)$, of $x$ is then

$$
g(x)=\int g_{2}(x, y) d y=\text { of } \frac{\sqrt{f(x)}}{y /|\Omega| d y=f(x) / 2|\Omega|}
$$

In order to have $g$ as a density function we should, by (3.29), have the identity

$$
1=\int g(x) d x=\int f(x) d x / 2|\Omega|=K / 2|\Omega|
$$

This implies that $K=2|\Omega|$ and hence $g(x)=f(x) / K$ and we have shown that the method works as prescribed. 
Sampling from the region $\Omega$ must often be done by some rejection technique. But by subdivision of $\Omega$ into smaller regions and using easily computable bounds, as described above, the efficiency of this procedure can be made as good as for many other sampling schemes. In fact, this method often leads to algorithms which are small and fast.

\subsubsection{Von Neumann-Forsythe's method}

In 1949, von Neumann proposed a method for generating samples from an exponential distribution only based on comparisons of uniform deviates. $H$ is method has then been modified to give samples from any distribution satisfying a first-order differential equation. We describe this general algorithm here.

Let $f(x)>0$ be defined for all $x \geq 0$ and satisfying the ODE

$$
f^{\prime}(x)+b(x) f(x)=0 \quad 0 \leq x<\infty
$$

where $b(x) \geq 0$.

Let

$$
B(x)=0 \int x b(t) d t
$$

and assume that

$$
C=0 \int^{\infty} \operatorname{Exp}(-B(x)) d x<\infty
$$

Then

$$
f(x)=\operatorname{Exp}(-B(x)) / C
$$

is the unique solution of (3.34) and (3.37) implies that $f$ is a density function of a non-negative random variable.

Given this function we prepare two tables of constants $\left\{q_{k}\right\}$ and $\left\{r_{k}\right\}$ $, k=0,1,2, \ldots, k$ in the following manner.

Let $q_{0}=0$ and for each $k=1,2, \ldots, k$ let $q_{k}$ be the largest possible value subjected to the two constraints

$$
\begin{aligned}
& q_{k}-q_{k-1} \leq 1 \\
& B\left(q_{k}\right)-B\left(q_{k-1}\right) \leq 1
\end{aligned}
$$

The sequence $\left\{r_{k}\right\}$ is given by

$$
r_{k}=\int^{q k} f(x) d x \quad \text { for } k=0,1,2, \ldots, k
$$

$K$ is chosen as the least index $k$ such that $r_{k}$ exceeds the largest represantable number less that 1 . 
We also introduce the functions

$$
G_{k}(x)=B\left(q_{k-1}+x\right)-B\left(q_{k-1}\right) \quad k=1,2, \ldots, k
$$

With these definitions and tables the algorithm for generating a sample $n$ from $f(x)$ is as follows:

1. [Chose interval] set $k=1$ and generate $\xi$

2. [Test] If $\xi \leq r_{k}$ then go to step 4

3. [Increase interval] set $k=k+1$ and go back to step 2 .

4. [Compute $\eta$ in selected interval] Generate another $\xi$ and set $w=\xi\left(q_{k}-q_{k}-1\right)$.

5. Set $t=G_{k}(w)$.

6. Generate $\xi_{1}$.

7. [Test] If $\xi_{1} \geq t$ then go to step 11.

8. [Trial continues] Generate $\xi$.

9. [Test] If $\xi<\xi$, then set $t=\xi$ and go back to step 6 .

10. [Reject the trial] Go back to step 4.

11. [Accept] Deliver $n=q_{k-1+w}$ as the sample.

The original von Neumann algorithm for the exponential distribution i.e. $b(x)=1$ is the same as the algorithm given above without steps $1-3$ and with a minor modification in step 4. In fact, we do not have to choose the interval separately because the probability of rejecting $w$ in step 10 is the same as the probability of rejecting an interval in step 1-3. The rejection in step 10 could then be used to signal that a change of interval should take place and a modification of step 4 so that we change interval when we pass it is all we need to do.

As a measure of the efficiency of the algorithm we could take the expected number of uniform deviates generated per sample. This expectation is given by the expression

$$
N^{\prime}=\sum_{n=1}^{\infty} N(k)\left(r_{k}-r_{k-1}\right)+1
$$

Here $N(k)$ is the expected number of uniform deviates generated in the interval $\left[q_{k-1}, q_{k}\right]$ i.e.

$$
N(k)=\left(d _ { k } + 0 \int ^ { d k } \operatorname { E x p } ( G _ { k } ( w ) ) d w / \text { of } \int ^ { d k } \operatorname { E x p } \left(-G_{k}(w) d w\right.\right.
$$

where $d_{k}=q_{k}-q_{k-1}$. 
If we calculate $N$ for the von Neumann algorithm we get the expected number of uniform deviates needed per sample as $1+e /(e-1) \simeq 4.30$.

\subsection{SPECIAL DISTRIBUTIONS}

There are a lot of different sampling schemes for many commonly occuring density functions. These are often simple and elegant schemes and are often optimized in some sense. A collection of many of the known schemes can be found in $[15,16]$. In this section we will just discuss sampling from the normal distribution. A number of various sampling methods for generating a sample from this distribution exists but the question of which one to use is very much dependent on whether we want to write the algorithm in a high-level programming language or in assembler code or if the memory requirements impose restrictions or not. We will discuss four techniques based on the methods given in the previous section and give some computing characteristics for them.

The normal distribution with mean $\mu$ and standard deviation $\sigma$ has the density function

$$
f(y)=\frac{1}{\sigma \sqrt{2 \pi}} \operatorname{Exp}\left(-(x-\mu)^{2} / 2 \sigma_{2}\right)
$$

However, for sampling purposes it is sufficient to use the standardized normal distribution i.e. $\mu=0$ and $\sigma=1$ in (3.43) since if $\eta$ is a sample from the standardized distribution then $n \sigma+\mu$ is a sample from (3.43). In the following we will only deal with the standardized distribution.

\subsubsection{Direct method}

We can not use the inversion formula (3.3) directly since we can not get the normal distribution function in a closed form. The "table method" could of course be used or we could approximate the distribution function with a rational approximation so that we could use (3.3). But if we investigate the joint distribution of two independent normal variables we observe that we can get an inverse in another way. This solution was first proposed by Box-Muller. Since we have two independent variables we get the joint density as the product of the individual densities i.e.

$$
f\left(y_{1}, y_{2}\right)=\operatorname{Exp}\left(-\left(y_{1}{ }^{2}-y_{2}{ }^{2}\right) / 2\right) / 2 \pi
$$

If we define the transformation

$$
\begin{aligned}
& y_{1}=\sqrt{\left(-2 \ln \xi_{1}\right)} \cos 2 \pi \xi_{2} \\
& y_{2}=\sqrt{\left(-2 \ln \xi_{1}\right)} \sin 2 \pi \xi_{2}
\end{aligned}
$$


where $\xi_{1}$ and $\xi_{2}$ are uniform deviates, then $y_{1}$ and $y_{2}$ are two independent normal deviates. This is easily verified if we look at a probability element $d \xi_{1} d \xi_{2}$ and transform it to a probability element in the $y_{1}-y_{2}$-space. We then get

$$
d \xi_{1} d \xi_{2}=|J| d y_{1} d y_{2}=f\left(y_{1}, y_{2}\right) d y_{1} d y_{2}
$$

where $J$ is the Jacobian.

\subsubsection{Composition-rejection method}

The composition-rejection method can be used to sample from the normal distribution in the following way. The selection of a sign at random is no greater problem so we may consider the distribution of the absolute value of a standard normal variable. We thus have the density function

$$
f(y)=\sqrt{(2 / \pi)} \cdot \operatorname{Exp}\left(-y^{2} / 2\right) \text { for } y \geq 0
$$

We then express $f(y)$ as

$$
f(y)=a_{1} f_{1}(y) g,(y)+a_{2} f_{2}(y) g_{2}(y)
$$

where the parameters and functions are given as

$$
\text { for } 0 \leq y \leq 1: \begin{aligned}
a_{1} & =\sqrt{(2 / \pi)} \\
f_{1}(y) & =1 \\
g_{1}(y) & =\operatorname{Exp}\left(-y^{2} / 2\right) \\
\text { and for } y>1: a_{2} & =\sqrt{2 \pi} \\
f_{2}(y) & =2 \operatorname{Exp}(-2(y-1)) \\
g_{2}(y) & =\operatorname{Exp}\left(-(y-2)^{2} / 2\right)
\end{aligned}
$$

\subsubsection{Ratio of uniform deviates}

We have the density function in (3.43) with $\mu=0$ and $\sigma=1$ and if we write $K=\sqrt{2 \pi}$ and $f(y)=\operatorname{Exp}\left(-y^{2} / 2\right)$ we can write it as (cf. 3.29)

$$
g(y)=k^{-1} \cdot f(y)
$$

The region $\Omega$ is then

$$
\Omega=\{(u, v): 0 \leq v \leq \operatorname{Exp}(v /(u \sqrt{2}))
$$

If we solve this relation, with $0 \leq u \leq 1$, we get

$$
v= \pm 2 u \sqrt{(-\ln u)}
$$


This relation is symmetric about the $v$-axis, each side representing the positive and negative part of the normal density function respectively. Thus it is sufficient to select points $(u, v)$ in the region $v \geq 0$ and then attaching a random sign to the deviate to get the normal sample. To increase the efficiency the region could be divided with triangles and rectangles (e.g. one subdivision could yield an efficiency of about $92 \%$ compared to $73 \%$ with one simple rectangle) and use easily computable bounds ( e.g. the need for log-evaluations in the acceptance test could be as little as one fourth of the tests.

\subsubsection{Von Neumann-Forsythe's method}

If we use the von Neumann-Forsythe's method with $b(x)=x$ then we get $B(x)=x^{2} / 2$ and $f(x)$ as in (3.47). Calculating the sequence \{qk\} gives us

$$
q_{0}=0 ; q_{k}=\sqrt{(2 k-1)} \quad k \geq 1
$$

and the $r_{k}{ }^{\prime} s$ are given by

$$
r_{k}=\operatorname{erf}\left(q_{k}\right) k \geq 1
$$

If we select $k=12$ we will generate normal deviates up to \pm 5 and the deviates will be truncated less than once in a million trials. If this is not enough we can choose a higher $K$. The average number of generated uniform deviates as given by $(3.41)$ is around 4.04 .

\subsubsection{Comparison of the methods}

To get a little more qualitative comparison of these four methods discussed above they were coded in FORTRAN and run on the CERN IBM $370 / 168$. Each routine was called 10000 times to produce normal deviates in a FORTRAN DO-loop. The average execution time, storage requirements and the mean number of used uniform deviates per call was recorded and are shown in rable 1 . In the direct method most of the execution time is spent on evaluation standard functions and we would not get any larger improvement in speed if we coded it in assembler code. But for the forsythe method a machine code version would probably run much faster. As expected the ratio method performs very well but needs a lot of storage. 


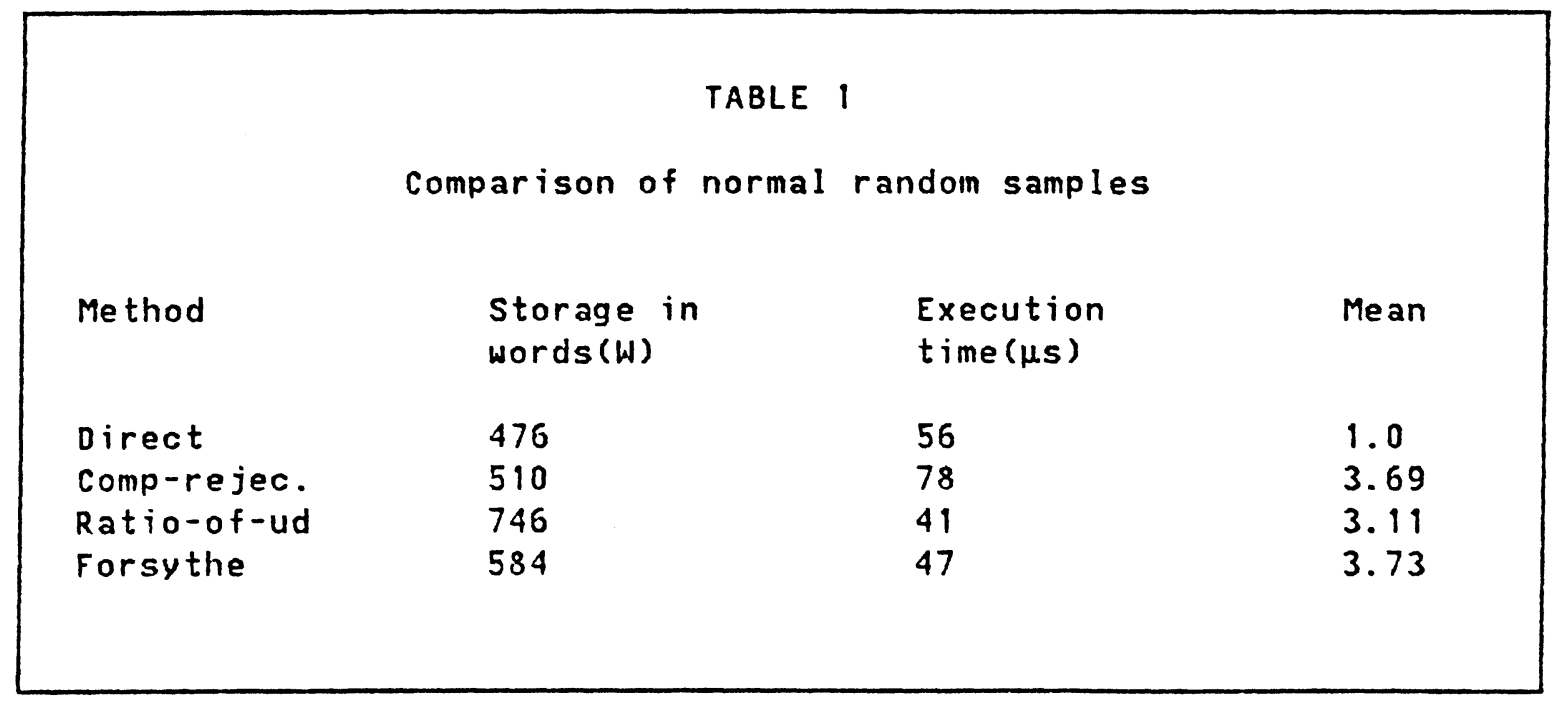

\subsection{FURTHER REFERENCES}

Forsythe's method is exposed in [18] and an extension to the method can be found in [1] and [36]. The ratio-of-uniform deviates method is described in [29] and [40] gives an implementation of sampling schemes for the normal and the gamma distributions.[2] compares the most common procedures for sampling from the exponential and from the normal distributions. In [8] the generation of samples subject to certain constraints are discussed. 
Chapter 4

PRINCIPLES OF MONTE CARLO CALCULATIONS

\section{1 INTRODUCTION}

So far we have been discussing how to generate uniform random numbers and how to transform them into the distributions we are interested in. In this chapter we will describe the principles of the Monte Carlo calculations and we will see how these random numbers are used. When we are studying the Monte Carlo methods we divide the methods into two classes:

1. Direct simulation of a probabilistic problem.

2. Simulation of a problem, direct or reformulated, that can be seen as a numerical integration.

The methods belonging to the first class are quite straightforward and have less theoretical interest than the second class methods. We will just give some examples of direct simulation and then we look at the properties of the methods in the second class.

\subsection{DIRECT SIMULATION}

Direct simulation could often be viewed as a random search in some specific lattice which in turn is nothing but a minimization of a suitable function subject to imposed restrictions. The method has been used in fields such as statistical tests (e.g. the student experiment), operations research and combinatorial problems.

We illustrate the method with a scheduling problem [38]. We are given $J$ jobs which each require processing on $M$ machines, each job passing from machine to machine in order. We want to decide in which order the jobs should be entered into the system so that some cost function is minimized. This function could of course be calculated for all permutations of the jobs but since this involves $J$ ! function evaluations this is only feasible for small $\mathrm{J}$. It is reasonable to suppose that if we, for some permutation of the $J$ jobs, have a small value of the cost a small perturbation of the order would not change the cost very much. If we define a distance between two permutations, e.g. the Hamming distance, the minimization could be made as follows:

First we choose a base permutation. Then we make a random selection of a permutation from the permutations within a given distance 
from the base permutation until we find one with lower cost. We then take this permutation as the new base and continue the process until no improvement has occurred for some time.

The efficiency of this procedure depends on many factors e.g. the choice of distance, how to perform the random selection and heuristic methods in determining a new base permutation. This example shows one of the typical features of direct simulation: A fairly simple general structure with a lot of heuristics and particular details.

We also mention a rather peculiar application of the Monte Carlo method. In [20] an analysis of the batting order in baseball is made with a Monte Carlo simulation. It is based on a quantification of the performance of each player and by a random selection of the outcome of the batting the different batting orders can be examined in terms of won/lost games.

\subsection{MONTE CARLO AS NUMERICAL INTEGRATION}

\subsubsection{Examples}

We start this section by giving two examples of calculations with the Monte Carlo method. Suppose that we are interested in knowing the area of the region $\Omega$ where (see Fig. 2)

$$
\Omega=\{(x, y): 0 \leq y \leq f(x)=\operatorname{Exp}(-1 / x)\}
$$

A very simple but immediate way to do this is to select points randomly in the rectangle $R=\{(x, y): 0 \leq x \leq 1,0 \leq y \leq 1 / e\}$ and then count the number of points which falls into the region $\Omega$. Say that we select in totally $m$ points and that $m^{\prime}$ of these are in the region $\Omega$. Then we feel quite sure of that the ratio $\mathrm{m}^{\prime} / \mathrm{m}$ is close to the ratio of the areas of region $\Omega$ to the region $R$ (i.e. close to $|\Omega| /|R|$ ) at least if we have chosen a large number of points $\mathrm{m}$. Thus we get an estimate of the area of $\Omega$ as

$$
|\Omega|=|R| \cdot m^{\prime} / m
$$

We made this calculation for $m=10000$ points, the points being chosen uniformly in $R$ i.e. $x_{i}=\xi_{i}$ and $y_{i}=\xi_{i} / e$, and we got the the area to $|\Omega| \cong 0.14815$. How accurate is this value? For the moment we do not know how to get an estimate of the error. Instead, we could try to find another way to calculate the area and see if we get the same result.

The area could be expressed as an integral i.e.

$$
|\Omega|=\int^{\prime} \operatorname{Exp}(-1 / x) d x
$$

Say that we do not know how to calculate this integral by analytical means. But we know some probability theory and we know that the expectation of a function with regard to a density function $p$ defined on the interval $[a, b]$ is 


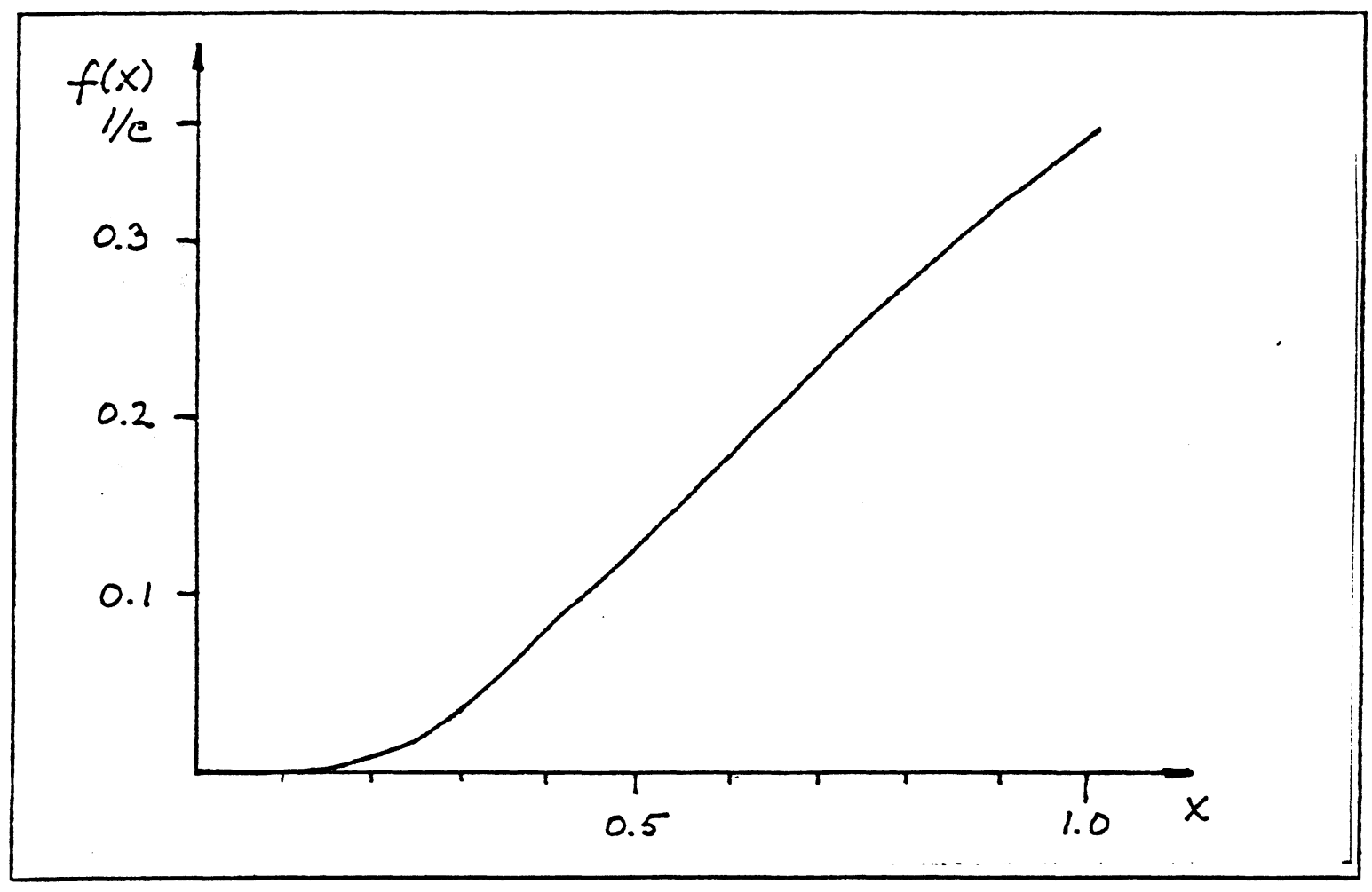

Figure 2: The function $\operatorname{Exp}(-1 / x)$

$$
E[f(x)]=\int_{a}^{b} f(x) p(x) d x
$$

and if we choose $p(x)$ as a uniform distribution on $[0,1]$ i.e. $p(x)=1$ then (4.4) is the same integral as (4.3). As an estimate of $|\Omega|$ we then could take the random variable

$$
x_{1}=\operatorname{Exp}\left(-1 / \xi_{1}\right)
$$

where $\xi_{1}$ is selected uniformly on $[0,1]$. If we then continue to choose independent random numbers $\xi_{i}$ we will get a set of independent and identically distributed random variables $x_{i}$. We then know that the mean of the $x_{i}$ will give us a better estimate of (4.3) than (4.5). If we select $m$ points $\xi i$ then the mean is

$$
(1 / m) \sum_{i=1}^{m} x_{i}=(1 / m) \sum_{i=1}^{m} \operatorname{Exp}\left(-1 / \xi_{i}\right)
$$

As in the previous example we made this calculation with $m=10000$ and we got the estimate of the area to $|\Omega| \simeq 0.14854$.

We now have two different values of the area. Which one is the most accurate? To answer that question we have to make a further investigation of what we have done. 


\subsubsection{Formulation}

A Monte Carlo calculation can be regarded as estimating the value of a multiple integral. If we sample a point $n$ from a distribution $F(x)$ defined on $\Omega$ then the random variable $g(n)$ is an estimator of the multiple integral in (4.7) since the expected value of $g$ is

$$
E[g(n)]=\int_{\Omega} g(t) d F(t)=\theta
$$

If we suppose that $g(x)$ is square integrable then we know that the variance of the estimator $g(n)$ is finite. We have

$$
\operatorname{Var}[g(n)]=\iint_{\Omega}|g(t)-\theta|^{2} d f(t)=\sigma^{2}<\infty
$$

We call $g(n)$ a primary estimator of $\theta$. If we sample $m$ points $n_{1}, n_{2}, \ldots, n_{m}$ independently from $F(x)$ then we can express a secondary estimator $g_{m}\left(n_{1}, \ldots, n_{m}\right)$ as the arithmetic mean of the values $g\left(n_{i}\right)$.

$$
g_{m}\left(n_{1}, \ldots, n_{m}\right)=(1 / m) \sum_{i=1}^{m} g\left(n_{i}\right)
$$

The expected value and the variance of $9 \mathrm{~m}$ is given by

$$
\begin{aligned}
& E\left[g_{m}\right]=\theta \\
& \operatorname{Var}\left[g_{m}\right]=\operatorname{Var}[g] / m=\sigma^{2} / m=\sigma_{m}^{2}
\end{aligned}
$$

By the law of large numbers we know that $E\left[g_{m}\right]$ and $\operatorname{Var}\left[g_{m}\right]$ converges to $\theta$ and 0 respectively with probability one (or in quadratic mean) as $m \rightarrow \infty$.

With resort to the central limit theorem we can now give an error estimate for the secondary estimator. The theorem asserts that the sum of $m$ independent and identically distributed random variables with mean $\theta$ and variance $\sigma^{2}$ are asymptotically normally distributed with mean $m \theta$ and variance $m \sigma^{2}$. This will give us an error bound, not an exact bound as we usually get in numerical analysis but we get a confidence interval for the estimate i.e. We can make assertions like "the estimate lies in this interval with a probability of p\%".

In our case the probability that our estimate of $\theta$ is far away from the exact value i.e. the probability that $\left|\left(g_{m}-\theta\right) / \sigma_{m}\right|>\alpha$ is given by

$$
\operatorname{Prob}\left(\left|\left(g_{m}-\theta\right) / \sigma_{m}\right|>\alpha\right)=2(1-\phi(\alpha))
$$

where $\phi(x)$ is the standard normal distribution.

The error we make in estimating the integral with $g_{m}$ is $\left|g_{m}-\theta\right|$. If we choose $\alpha=2$ in (4.11) we see that $\left|g_{m}-\theta\right|>2 \sigma_{m}$ with probability asymptotically equal to $0.0455 \mathrm{i} . e$. the probability that the error is greater than two standard deviations is around $5 \%$. 
We also see that the sampling error, $\left|g_{m}-\theta\right|$, depends on $\sigma_{m}$. Thus, the convergence in probability of $g_{m}$ to $\theta$ is of the order $0(1 / \sqrt{m})$. This is a quite slow convergence especially if we compare it with other methods for numerical integration in few dimensions. E.g. for the simpson rule in one dimension the convergence is of the order $O\left(m^{-4}\right)$. But in the Monte carlo method we are always sure to have the convergence speed no matter in how many dimensions we are integrating and no matter how ill behaved the integrand is. The lesson to be learned is: Use Monte Carlo only if there is no other better method to solve our problem and do not use it as a "passepartout".

From above we conclude that the variance $\sigma_{\mathrm{m}}^{2}$ is a measure of the error in the estimate $g_{m}$ of $\theta$. However, we almost never know the variance explicitly so it is useful to have an estimator of it. An unbiased estimator of the variance is given by

$$
s_{m}^{2}=\frac{1}{m-1}\left(\sum_{i=1}^{m}\left(g\left(n_{i}-g_{m}\right)^{2}\right)=\frac{1}{m-1}\left(\sum_{i=1}^{m} g\left(n_{i}\right)^{2}-\left(\sum g\left(n_{i}\right)^{2} / m\right)\right.\right.
$$

As we have seen, it is the variance that reflects the sampling error. This fact could be taken as a mean of constructing a measure of efficiency of various Monte Carlo methods. The measure should also reflect that different method involve different amount of work. Therefore, if $\sigma_{1}{ }^{2}$ and $\sigma_{2}{ }^{2}$ is the variances of two different methods which each require $n_{1}$ and $n_{2}$ units of computing time, we define the relative efficiency of method 2 to method 1 as

$$
\left(n_{1} / n_{2}\right) \cdot\left(\sigma_{1}^{2} / \sigma_{2}^{2}\right)
$$

Here we have two terms: the labour ratio $n_{1} / n_{2}$ and the variance ratio $\sigma_{1}{ }^{2} / \sigma_{2}^{2}$. The former depends on the method and on the computer on which we run our problem and the latter is function of the problem and of the different methods used.

In order to increase the efficiency and to decrease the sampling error we are thus faced with the problem of decreasing the variance. There are a lot of standard methods for variance reduction which have a high efficiency compared to the crude Monte Carlo method, and we will discuss several of them in the next chapter.

If we look at the examples discussed above we see that in the first example we estimated

$$
\theta=\int_{R} h(x, y) d x d y
$$

where $\quad h(x, y)=0$ if $f(x)=\operatorname{Exp}(-1 / x)<y$

$$
=1 \text { if } f(x)=\operatorname{Exp}(-1 / x) \geq y
$$

and we estimated the integral with 


$$
g_{m}=-\sum_{m}^{m} h\left(n_{2} i-1, n_{2} i\right)=m^{\prime} / m
$$

We say that we have a hit or miss if the point is in the region $\Omega$ or not and because of that we call this method for hit-or-miss Monte Carlo. This procedure is a sampling from a binomial distribution with probabil$i$ ty of hit $p=e \theta$. The variance is then

$$
\sigma_{m}^{2}=e \theta(1-e \theta) / m
$$

The second example is an example of what is called crude Monte Carlo, and $i t$ is an example of the formalism described above. From (4.8) we see that the variance of the estimator is

$$
\sigma_{m}^{2}=\frac{1}{m}\left(0 \int^{\prime} \operatorname{Exp}(-2 / t) d t-\left(\text { of } \int^{\prime} \operatorname{Exp}(-1 / t) d t\right)^{2}\right)
$$

If we calculate the integral in (4.7) and the variance in (4.8) for the test function $f(x)=\operatorname{Exp}(-1 / x)$ we get the value of $\theta \simeq 0.148496$ and the variance of $\operatorname{Var}[\theta] \simeq 1.5483 \cdot 10^{-2}$

We use (4.14) to calculate the variance of the hit-or-miss method and we get that the variance ratio of the crude to the hit-or-miss method is around 0.065 . The labour ratio is around 1 so we conclude that in order to get the same accuracy in the hit-or-miss as in the crude method we have to use around 16 times more samples in the hit-or-miss sampling. The crude sampling has always a lower variance than the hit-or-miss sampling as we can see by looking at the difference between the two variances. If we let $a h$ stands for $h i t-o r-m i s s$ and $a c$ for crude then the difference is

$$
\begin{aligned}
\sigma_{h}^{2}-\sigma_{c}^{2} & =\theta(1-\theta) / m-(1 / m) \int f^{2}(x) d x+\theta^{2} / m= \\
& =(1 / m) \int f(x)(1-f(x)) d x
\end{aligned}
$$

We see that for all functions $f \geq 0$ this difference will always be positive.

We can, for both methods, calculate a $95 \%$ confidence interval for the estimated integral, $\theta$, by using the sample variance in (4.12). We compare the values with the correct value and with a value calculated with a 10-point Gaussian quadrature formula.

This is an example of a general rule for Monte Carlo calculations: If, at any point of a calculation, we can replace an estimate by an exact value, we reduce the variance, and consequently the sampling error, in the final result. What we have done in the passage from hit-or-miss to crude sampling is equivalent to replacing the function $h(x, n)$ by its exact expectation $f(x)$. 
TABLE 2

crude and hit-or-miss sampling

\begin{tabular}{|l|c|c|c|}
\hline Method & $\theta$ & $\operatorname{Var}[\theta]$ & Abs. error \\
\hline Hit-or-miss & $0.14815 \pm 0.0077$ & $1.47 \cdot 10^{-3}$ & 0.00035 \\
\hline Crude & $0.14854 \pm 0.0025$ & $1.54 \cdot 10^{-4}$ & 0.00004 \\
\hline Gauss quad. & 0.148495 & 0.000002 \\
\hline
\end{tabular}

\subsubsection{Numerical quadrature}

When we are talking about numerical quadrature we mean the calculation of an integral by the means of a sum. In general we have a formula like

$$
\int_{\Omega} f(x) d x=\Sigma w_{i} f\left(x_{i}\right)
$$

where the weights $w_{i}$ depends on the chosen point set $\left\{x_{i}\right\}$. We then determine the point set $\left\{x_{i}\right\}$ and the weights $w_{i}$ for a given class of functions by solving the integral for some members in the function class and then solving the resulting equations in $x_{i}$ and $w_{i}$. Then we can use the formula (4.18) for this function class without need to recalculate the points and the weights. We also get a precise error estimate often expressed in the derivatives of the function.

In the Monte carlo method we use the formula (4.18) with $w_{i}=1 / m$ and the $m$ points $x_{i}$ are chosen at random. We can, in principle, integrate every function with this choice of weights and points by choosing sufficiently many points.

The error estimates in the Monte Carlo method depends on the variance But since we are dealing with random number generators which do in fact produce deterministic sequences, objections have been made to the use of statistical arguments for justifying the error bounds. These objections has led to a new method, the quasi-Monte Carlo method. It resembles the Monte Carlo method in that it uses the same weights, $1 / m$, in $(4.18)$. But it reject the use of random numbers. However, we retain the property of these numbers of being uniformly spread over an interval by using equidistributed points $x_{i}$. We then have points with known properties and we can express the error in terms of discrepancy and variation (see appendix B). The quasi-Monte Carlo method is of great theoretical interest 
and several results on equidistributed sequences and on discrepancies has emerged. It is, however, far from being completely understood and a lot of work remains.

\subsubsection{Characteristics of the Monte Carlo method}

We summarize the most important characteristics of the Monte Carlo method in the seven points below.

1. The Monte Carlo estimate (4.9) is consistent i.e. it converges to the true value, if the variance of the integrand is finite.

2. The estimate is asymptotically normal distributed.

3. The choice of primary estimators could widely affect the efficiency of the method. The primary estimators need not be unbiased and different estimators can be used during the course of a computation.

4. The variance of the estimate is given by $\sigma^{2} / \mathrm{m}$, where $m$ is the sample size. This implies that the convergence speed in all dimensions is of the order $0\left(m^{-1 / 2}\right)$.

5. The only restriction we impose on the integrand is that is has finite variance. In particular we do not have any restrictions on the smoothness of the integrand.

6. The method is most suitable for integrating in many dimensions and/or integrating difficult functions.

7. The method should only be used on a problem which we do not know any better way to solve it i.e. We should investigate the problem carefully.

\section{4 FURTHER REFERENCES}

General text books on the Monte Carlo method is [26] and [43] and [27] and [24] are two good survey papers. In [21] one finds some applications of the method to probabilistic problems. General theory for multidimensional integration could be found in [47] and the paper [23] is a survey of the used methods. The quasi-Monte Carlo method is exposed in [51]. An implementation of the Monte Carlo method for multiple integration is described in [22]. 


\author{
Chapter 5 \\ VARIANCE REDUCTION
}

\title{
5.1 INTRODUCTION
}

The error in a Monte Carlo calculation depends on the variance and the sample size according to

$$
\operatorname{Error}^{2} \simeq(\operatorname{Var}[f] / m)
$$

If we want to get a higher accuracy in our result we have two possibilities: Either increase the sample size $m$ or decrease the variance. The former is an easy change of some parameters whilst the latter requires a careful study of the problem. The sample size is bounded upwards by the speed of the computer and by the cost to run the problem and usually we already use the largest acceptable sample size. It then remains to reduce the variance. This can be done by some general methods applicable to almost all problem types or by some tricky and intricate procedures especially developed for some smaller class of problems or for the problem at hand. Here we will discuss the general methods and in the next chapter we will show some tricks used in particle transport calculations.

\subsection{STRATIFIED SAMPLING}

A way that seems quite obvious to improve the accuracy is to divide the integration interval into several pieces and apply the crude method to each piece. If the interval is $[0,1](s a y)$ then we specify division points $a_{j}$ such that $0={ }_{0}<a_{1}<\ldots<a_{k}=1$. Then we make the crude sampling with $m_{j}$ points in each subinterval $\left[a_{j}-1, a_{j}\right]$ and as the estimate of the integral we take

$$
\theta=\sum_{j=1}^{k} \sum_{i=1}^{m_{j}}\left(\alpha_{j}-\alpha_{j}-1\right) / m_{j} f(n)
$$

where $n$ is uniformly distributed in the interval $\left[a_{j-1}, a_{j}\right]$.

This estimator is unbiased and has variance

$$
\operatorname{Var}[\theta]=\sum_{j=1}^{k}\left(a_{j}-a_{j-1}\right) / m_{j} \int_{\alpha_{j-1}}^{\alpha_{j}} f(x)^{2} d x-\sum_{j=1}^{k} 1 / m_{j}\left(\int_{\alpha_{j-1}}^{\alpha_{j}} f(x) d x\right)^{2}
$$


This variance is not necessarily less than the variance for the crude estimator (4.9) with $m=\Sigma m$ but if we choose the points $\alpha_{j}$ carefully we will get a reduction. This happens if the difference between the means in various intervals is greater than the variation of $f$ within the intervals. This can be seen if we look at the difference between the variances for the crude method $\left(\sigma_{c}^{2}\right)$ and the stratified sampling $\left(\sigma_{s} t^{2}\right)$. We have

$$
\sigma_{c}^{2}-\sigma_{5} t^{2}=\sum_{i<j}\left|\theta_{i}-\theta_{j}\right|^{2}-(1 / m) \sum_{j}\left(1-\left(m / m_{j}\right)\left(a_{j}-a_{j}-1\right)\right) \int_{\alpha_{j-1}}^{a_{j}} f^{2} d x
$$

where $\theta_{i}=\int_{\alpha_{j-1}}^{a_{j}} f d x$

The division of the interval could be made in different ways. A good way is to choose the points so that the variation of $f$ is the same in each interval. This is often difficult to do since we must know the variance of $f$ in the different pieces. We can make a simpler division and still be sure of getting a variance reduction (even though it may be arbitrary small). The partition of the interval into equal parts and the use of equal sample sizes will give us this reduction. From (5.4) we see that the improvement in the variance is

$$
\sigma_{c}^{2}-\sigma_{5} t^{2}=\sum_{i<j}\left|\theta_{i}-\theta_{j}\right|^{2} \geq 0
$$

If the division points are given then the best way to spread the total number of sampling points $m=\Sigma m_{j}$ is to choose the $m_{j}{ }^{2}$ to be proportional to

$$
\left(a_{j}-a_{j}-1\right) \int f(x)^{2} d x-\left(\int f(x) d x\right)^{2}
$$

The test example was solved with stratified sampling and we used an equal partition of the interval into four pieces and varied the sample sizes. With the total sample size fixed, $m=10000$, we made two calculations: one with equal sample sizes $m_{j}=2500$ and one with sample sizes chosen according to eqn.(5.6). The result is shown in table 3

If we compare the theoretical results for the stratified sampling with the crude-Monte Carlo for our test problem we get the variance ratios to 17.7 for the equal sample size and to 14.4 for the other. The labour in stratification is about the same as in the crude method if we assume that it is the function evaluations that are time consuming. The efficiency gain is then about 15 times over the crude Monte Carlo. As a general rule the efficiency of stratified sampling increases as the square of the number of division points $\alpha_{j}$. 
TABLE 3

stratified sampling

\begin{tabular}{|c|c|c|c|}
\hline Method & $\theta(95 \%)$ & $\operatorname{Var}[\theta]$ & Abs. error \\
\hline Equal samples & $0.14879 \pm 0.0006$ & $9.02 \cdot 10^{-4}$ & 0.00029 \\
\hline $\begin{array}{c}m_{j}=100,1310 \\
=3340,5250\end{array}$ & $0.14902 \pm 0.0007$ & $1.09 \cdot 10^{-3}$ & 0.00052 \\
\hline
\end{tabular}

\subsection{CONTROL VARIATES}

In stratified sampling our philosophy is that in the regions where our function contributes more to the variation we try to get a more accurate value by making comparatively more samples in that region. Another idea to reduce the variance is to modify our integral so that we perform the Monte carlo calculation on a function with smaller variance. This is what we do in control variate sampling. We approximate the function with an easy function $\phi(x)$ and as the estimate we take

$$
\theta=\int \phi(x) d x+\int(f(x)-\phi(x)) d x
$$

The easy function should be chosen so that we can calculate the first integral in (5.7) by analytical means. Then we calculate the second integral with crude Monte Carlo. The control variate in the estimator (5.7) is the easy function $\phi$. The estimator $\theta$ is clearly unbiased and its variance is

$$
\operatorname{Var}[\theta]=\operatorname{Var}[f]+\operatorname{Var}[\phi]-2 \operatorname{Cov}[f, \phi]
$$

Thus if $\phi$ is positively correlated to $f$ and $2 \operatorname{Cov}[f, \phi]>\operatorname{Var}[\phi]$ then we get a variance reduction in comparision with the crude estimator.

We could look at this method in another way. Say that we want to solve a complicated problem and that we know an analytical solution to a simpler version of the problem. Then we could use the correlation between the problems to solve the complicated one. We do this by making a simultaneous Monte Carlo calculation of both problems using the same random numbers. In the notation above $\theta$ is our complicated problem and $\phi$ is the known solution. The estimate is then

$$
\theta=E[\phi]+E[f]-E\left[\phi^{\prime}\right]
$$

where $E[f]$ and $E\left[\phi^{\prime}\right]$ is the Monte Carlo estimates. 
In the test example we choose a simple function which follows the variations of $f$. The simplest choice is just a linear function and we take

$$
\phi(x)=e^{-1} x \quad \text { for } x \in[0,1]
$$

The calculations with $m=10000$ points gives the estimate 0.14915 and the variance $6.73 \cdot 10^{-4}$. A $95 \%$ confidence interval for $\theta$ is then $0.14915 \pm 5.2 \cdot 10^{-4}$. The theoretical variance is $6.83 \cdot 10^{-4}$ and the variance ratio is therefore 22.7. The control variate method requires about two times more work so with labour ratio 2 the efficiency ratio is about 11. We could also choose a more close approximation of $f$ as for example a piecewise linear approximation. A calculation was made with the control variate

$$
\begin{array}{rlrl}
\phi(x) & =0 & & \text { if } x \leq 0.2 \\
& =e^{-1}(5 x-1) / 4 & \text { if } 0.2 \leq x \leq 1
\end{array}
$$

With the same number of sampling points this choice gives the estimate $0.14865 \pm 1.37 \cdot 10^{-4}(95 \%)$ and variance $4.71 \cdot 10^{-5}$. The variance ratio is now 320 so the relative efficiency is around 160 .

\subsection{IMPORTANCE SAMPLING}

Instead of approximating the function $f$ and thus getting a lower variance we could concentrate the distribution of the sampling points in the parts which are of most importance i.e. contributes most to the variance. This could be done in the following way:

We have the identity relation

$$
\theta=\int f(x) d x=\int(f(x) / \phi(x)) \phi(x) d x=\int(f(x) / \phi(x)) d \Phi(x)
$$

for any functions $\phi$ and $\phi$ satisfying

$$
\Phi(x)=0 \int x \phi(t) d t
$$

If we restrict $\phi$ to be a positive-valued function and normalize so that

$$
\int \phi(x) d x=1
$$

then $\Phi$ is a distribution function. If we sample points $n$ from this distribution we see from (5.12) that $f(n) / \phi(n)$ has expectation $\theta$ and variance

$$
\operatorname{Var}[\theta]=\int((f(x) / \phi(x))-\theta)^{2} d \Phi(x)
$$

The bias introduced when we sample from $\phi(x)$ is compensated for by taking $f / \phi$ as the estimator instead of $f$. 
The best choice of function $\phi$ in the case when $f$ is also a positivevalued function is to take $\phi$ proportional to $f$ i.e. $\phi=c f$. Then (5.14) implies that $c=1 / 6$ and $(5.15)$ then shows that the variance for this $\phi$ is zero. But this choice is of course not feasible since we have to know $\theta$ to get the importance function $\phi$ and since it is $\theta$ we are trying to calculate we are back to the initial problem. This could, however, be a guideline of how to choose the function $\phi$. We are scoring the values of $f / \phi$ and we get a small variance if this quotient is as constant as possible. The importance function $\phi$ should therefore mimic $f$.

We demonstrate the method with the test function. With the same choice of function as in the first example of the control variate method $(5.10)$ we get the distribution function as

$$
\Phi(x)=x^{2}
$$

To sample from (5.16) it is easiest to take the sample point $n$ as $\max \left(\xi_{1}, \xi_{2}\right)$ where $\xi_{i}$ are uniform samples. A calculation with 10000 samples gave the results $\theta=0.14810$ and variance $=2.48 \cdot 10^{-7}$. A comparison of the true variances shows that the variance ratio to the crude method is 6.5 and the labour ratio is around 2. The efficiency gain is therefore about 3 .

Importance sampling and control variates are closely related to each other in that they approximate the function $f$ with an easy function $\phi$. We could examine which of the two methods is the best for a given easy function $\phi$. Let the constant $C$ be

$$
c=\int \phi(x) d x
$$

Then the difference between the variances is (cvecontrol variate, im =importance)

$$
\begin{aligned}
& \operatorname{Var}\left[\theta_{c v}\right]-\operatorname{Var}\left[\theta_{i m}\right]= \\
& =\int(f-\phi)^{2} d x-(\theta-c)^{2}-c \int f^{2} / \phi d x+\theta^{2}= \\
& =\int(f-\phi)^{2} d x-c \int(f-\phi)^{2} / \phi d x=\operatorname{Cov}\left[(f-\phi)^{2} / \phi, \phi\right]
\end{aligned}
$$

From this we see that if the approximation of $f$ by $\phi$ is "absolutely uniform" (i.e. $|f-\phi|$ is approximately constant for all values of $|\phi|$ ) then the control variate sampling is preferred while if the approximation is "relatively uniform" (i.e. $|f-\phi|$ is approximately proportional to $|\phi|$ ) the importance sampling is more efficient. 


\subsection{ANTITHETIC VARIATES}

The variance reduction we obtain when we use control variates is due to the positive correlation between the function $f$ and the easy function $\phi$. In the antithetic method we try to find an estimator which has the same expectation as the crude estimate and has a strong negative correlation to $i t$. We then use a linear combination of these estimators as the estimator. The term antithetic variate is used to describe a set of estimators which mutually compensate each other's variations.

In the case when $f$ is a monotonic function we have an immediate estimator. Since $1-x$ is uniformly distributed in $[0,1]$ if $x$ is then the primary estimator

$$
\theta=1 / 2(f(n)+f(1-n))
$$

is unbiased and has variance

$$
4 \operatorname{Var}[\theta]=\operatorname{Var}[f(x)]+\operatorname{Var}[f(1-x)]+2 \operatorname{Cov}[f(x), f(1-x)]
$$

Now if $f(x)$ is monotonically increasing (decreasing) then $f(1-x)$ is monotonically decreasing (increasing) and the covariance is then negative.

If we combine the stratified sampling with the antithetic variates we arrive at a slightly more general estimator. We take $k=2, \alpha_{j}=\alpha$ and $m_{1}=m_{2}=m$ in (5.2) and then we have

$$
\theta=-\sum_{m=1}^{m}\left(a f\left(a \xi_{i 1}\right)+(1-a) f\left(a+(1-a) \xi_{i_{2}}\right)\right)
$$

where $\xi_{i 1}$ and $\xi_{i 2}$ are uniformly distributed in the interval $[0,1]$. We can now introduce a functional dependence between $\xi_{i 1}$ and $\xi_{i 2}$. If we for example choose $1-\xi_{i_{2}}=\xi_{i_{1}}=\xi_{i}$ then we get the estimator

$$
\theta=-\sum_{m}^{1} \sum_{i=1}\left(a f\left(a \xi_{i}\right)+(1-a) f\left(1-(1-a) \xi_{i}\right)\right)
$$

which for $a=0.5$ is essentially equal to (5.19). The variance of $\theta$ in (5.22) is a function of $a$ and we could try to find an a for which the variance is minimized. This is usually a difficult task but if the function is monotonic we can as a rule of thumb choose a as the root of

$$
f(a)=(1-a) f(1)+a f(0)
$$

The estimators (5.19) and (5.22) with a determined by solving (5.23) were tested on the test problem. With the usual number of sampling points the first estimator gave $\theta=0.14872 \pm 3.2 \cdot 10^{-4}(95 \%)$ and the second gave $\theta=0.14854 \pm 1.2 \cdot 10^{-4}$. The variance ratios are 67 and 237 respectiveIy and the labour ratio is once again about 2 so we have an efficiency ratio of 34 and 120 respectively. It is notable that the change from $a=0.5$ to $a=0.5456$ gives this remarkable change in the variance ratio. 
Usually it is easier to find a negatively correlated, unbiased estimator of the function $f$ than it is to find a "good" importance function or a satisfactory control variate. Due to this fact the antithetic variates seems to be more efficient in practice. However, the method is very difficult to use when the function $f$ is very irregular e.g. in evaluating a multidimensional integral when the integrand varies rapidly in every direction.

\subsection{REGRESSION METHODS}

In order to reduce the variance we could also make use of the theory of multiple linear regression analysis. Suppose that we have several unknown estimands $\theta_{1}, \theta_{2}, \ldots, \theta_{k}$. We then form the estimators $t_{1}, t_{2}, \ldots, t_{n}$ $(n \geq k)$ as the linear combination

$$
t_{i}=x_{i} \theta_{1}+\ldots+x_{i k} \theta_{k} \quad i=1, \ldots, n
$$

where the $\left\{x_{i j}\right\}$ are known.

We want to estimate $\theta=\left(\theta_{1}, \ldots, \theta_{k}\right)$ and the regression theory then gives us the minimum-variance, unbiased estimator as

$$
t^{\prime}=\left(x^{t} v-1 x\right)-1 x^{t y-1} t
$$

where $x$ is the nxk matrix $\left\{x_{i j}\right\}, V$ is the nxn covariance matrix and $t$ is the $n \times 1$ column matrix $\left\{t_{i}\right\}$. Here we know everything but the covariance matrix $v$. The relations $(5.24)$ can be written in matrix notation as

$$
t=x \theta
$$

and the estimator $t^{\prime}$ is a linear function of $t$. This implies that if we look at (5.25) with a different covariance matrix $W$ the expectation of the estimator is

$$
E\left[t_{W^{\prime}}\right]=\left(x^{t_{W}-1} x\right)^{-1} x^{t_{W}-1} E[t]=\left(x^{t_{W}}-1 x\right)^{-1} x^{t_{W}-1} x \theta=\theta
$$

and so the estimator is still unbiased. But we do not have any minimumvariance estimator any longer. However, if we can get a good approximation of $v$ we can use the estimator $t_{w^{\prime}}$. The variance of this estimator is $\left(x^{t} w-1 x\right)^{-1}$ and the first-order errors in $w$ will only lead to secondorder errors in the estimate $t_{w^{\prime}} i . e$. our confidence interval for $t_{w^{\prime}}$ will be larger.

The calculations are then organized as follows: We make $m$ independent sets $\left\{t_{1 j}, t_{2 j}, \ldots, t_{n j}\right\}$ of estimates of the $t_{i}{ }^{\prime} s$. The estimates of the $t_{i}{ }^{\prime} s$ and the elements of the covariance matrix $w$ is then 


$$
\begin{aligned}
& E\left[t_{i}\right]=\sum_{j=1}^{m} t_{i j / m} \quad i=1, \ldots, n \\
& w_{i j} \quad=\sum_{1=1}^{m}\left(t_{i 1}-E\left[t_{i}\right]\right)\left(t_{j 1}-E\left[t_{j}\right]\right) /(m-1) \\
& \quad \text { for } i, j=1,2, \ldots, n
\end{aligned}
$$

The covariance matrix of the estimator $t_{w^{\prime}}$ is then

$$
\operatorname{Var}\left[t_{w^{\prime}}\right]=\left(x^{t} w^{-1} x\right)-1 / m
$$

The regression method exploits the correlation between the estimators and thereby reduces the errors in the result. It is even safe to use this method if there is no correlation for the price of a little more labour. The method can be applied in many different Monte Carlo situations. One typical situation is the combination of antithetic variates and regression. If we look at eqn. (5.19) we see that it could be written as a regression estimator. Let e.g. $t_{1}=f(x)$ and $t_{2}=f(1-x)$ and $\theta_{1}=E[f(x)]=E[f(1-x)]$. Then the estimate of $\theta_{1}$ is the estimator $t_{w}{ }^{\prime}$ with $x_{11}=x_{12}=1$.

Let us consider our test example. Take as the estimators (5.22) with $a=0.5$ and $\alpha=0.55$ i.e.

$$
\begin{aligned}
& t_{1}=0.5 f(0.5 \xi)+0.5 f(1-0.5 \xi)=\theta=1 \cdot \theta_{1} \\
& t_{2}=0.55 f(0.55 \xi)+0.45 f(1-0.45 \xi)=\theta=1 \cdot \theta_{1}
\end{aligned}
$$

Then $k=1, n=2$ and $x^{t}=(1,1)$. We made 10000 samples and we obtained the estimates $t_{1}^{\prime}=0.14828, \quad t_{2}^{\prime}=0.14944$ and the estimate of the covariance matrix $w$ was $w_{11}=2.57 \cdot 10^{-4}, w_{22}=3.00 \cdot 10^{-3}$ and $w_{12}=w_{21}=-6.89 \cdot 10^{-4}$. The estimate of $\theta$ was 0.14852 and with a $95 \%$ confidence interval we got the value $\theta=0.14852 \pm 0.00016$.

\subsection{ORTHONORMAL FUNCTIONS}

Orthonormal functions can be used to reduce the variance by approximating the function $f$ with a truncated fourier series provided that we have an appropriate basis of orthonormal functions. Suppose that the function $f(x)$ is defined in a k-dimensional space $\Omega_{k}$ and that we have a set of functions $\phi_{1}, \ldots, \phi_{n}$ in $\Omega_{k}$, orthonormal with respect to the weight function $w(x)$. We approximate $f$ by a linear combination of the functions $\phi_{i}$ i.e.

$$
f(x)=\sum_{i=1}^{n} a_{i} \phi_{i}(x)
$$


If we determine the variables $a_{i}$ as the solution of the system of equations

$$
\sum_{i=1}^{n} a_{i} \phi_{i}\left(x_{j}\right)=f\left(x_{j}\right) \quad j=1, \ldots, n
$$

with the points $x_{j}$ distributed according to the density function

$$
p_{n}\left(x_{1}, \ldots, x_{n}\right)=w\left(x_{1}\right) \cdots w\left(x_{n}\right) \phi\left(x_{1}, \ldots, x_{n}\right)
$$

where $\phi\left(x_{1}, \ldots x_{n}\right)=\operatorname{det}^{2}\left\|\phi_{j}\left(x_{i}\right)\right\| / n$ !

Then for $m=1,2, \ldots, n$ we have the relations

$$
\text { (i) } E\left[a_{m}\right]=A_{m}=\int_{\Omega_{k}} w(x) f(x) \phi_{m}(x) d x
$$

$$
\text { (ii) } \begin{aligned}
\operatorname{Var}\left[a_{m}\right] & =\int_{\Omega_{k}} w(x) f^{2}(x) d x-\sum_{i=1}^{n} A_{i}{ }^{2}= \\
& =\inf \int_{\Omega_{k}} w(x)\left[f(x)-\sum_{i=1}^{n} a_{i} \phi_{i}(x)\right]^{2} d x
\end{aligned}
$$

We see that (5.33) gives a set of primary estimators for the fourier coefficients in (5.32) and we can use them to estimate integrals of the type (i) above. From (ii) we see that we may get a variance reduction if we have chosen the basis appropriately and that we get a zero variance if $f$ lies in the space spanned by the basis functions $\phi_{i}(x)$.

The main difficulty with this method is the sampling from the density function $p_{n}$. This is ingeneral a very difficult task but, still, it might be worth trying this method if he complexity of the problem warrants the extra labour required to generate each estimate. It is notable that the sampling method depends on the region $\Omega_{k}$, the orthonormal functions $\phi_{i}$ and the weight function $w(x)$ and not on the function $f$ itself. Thus, if we want to integrate many different functions in the same region and with the same basis functions we gain a lot of work. timate

We look at an example in one dimension $(k=1)$. Say that we want to es-

$$
\theta=0 \int^{1} g(x) d x
$$

and that we use just one orthonormal function $\phi(x)$ with weight $w(x)=1$. The density function $(5.34)$ is then $p(x)=\phi(x)^{2}$ and the estimator, $a_{1}$, of the integral (5.37) is

$$
a_{1}=f(n) / \phi(n)=g(n) /(\phi(n))^{2}
$$

where $g(x)=f(x) \phi(x)$. 
The choice $\phi(x)=1$ is then simply the crude Monte Carlo method. If we know a little bit more about the functiong we could make a cleverly choice of base. Let $h(x)$ be defined as

$$
h(x)=g(x)-(1-x) g(0)-x g(1)
$$

Then we know that $h(0)=h(1)=0$ and the estimate, $\theta$, of the function $g$ is

$$
\theta=\int^{1} h(x) d x+\int_{0}^{1}(h(x)-g(x)) d x=\theta^{\prime}+0.5(g(0)+g(1))
$$

where $\theta^{\prime}=0 \int^{1} h(x) d x$

We choose a function $\phi$ with the same properties as $h$ e.g. take $\phi=\sqrt{(6 \times(1-x))}$. Then by $(5.37)$ we get the estimator

$$
\theta=(g(0)+g(1)) / 2+(g(n)-(1-n) g(0)-n g(1)) / 6 n(1-n)
$$

where $n$ has density $6 \times(1-x)$. The sampling point $n$ is easily obtained if we observe that if $\xi_{1} \geq \xi_{2} \geq \xi_{3}$ where $\xi_{i}$ is three independent uniform deviates then $\xi_{2}$ has the desired density. This latter estimator was tested on the function $g(x)=\operatorname{Exp}(-1 / x)$. With 10000 sample points we obtained the result $\theta=0.14864$ and $\operatorname{Var}[\theta]=4.72 \cdot 10^{-4}$.

\subsection{CONDITIONAL MONTE CARLO}

Conditional Monte Carlo is not a variance reduction techniquie in its own but it can be seen as a form of importance sampling and we include it here as an example of that technique. The conditional Monte carlo problem is defined as follows: Let $z$ be a vector in an k-dimensional space $z$ which has an unconditional density function $h(z)$. The problem is then to determine the expected value $\theta$ of an arbitrary function $p(z)$ under a condition $n(z)=y_{0}$, a surface in the $z$-space, i.e.

$$
\theta=\int p(z) h\left(z \mid \eta(z)=y_{0}\right) d z
$$

The sampling from the conditional density function $h\left(z \mid n(z)=y_{0}\right)$ is of ten very difficult and it is desirable to have a method that allows one to sample from the density function $h(z)$.

To be able to sample from $h(z)$ we introduce a transformation $\xi(z)=x$. The only restriction on the transformation $\xi$ is that there should be a 1:1 correspondence between the intersection of the two surfaces $x$ and $y$ and the point $z$ i.e. a $1: 1$ correspondence between the point $(x, y)$ in the product space $X \cdot Y$ and the point $Z$ in the space $Z$. We can then write

$$
h(z) d z=h(x, y) d z=h(x, y) d x d y / J(x, y)=f(x, y) d x d y
$$

where $J(x, y)=|d x d y / d z|$ is the Jacobian of the transformation. The conditional density of $x$ for a $g$ iven $y=y_{0}$ is then 


$$
f\left(x \mid y_{0}\right)=\frac{f\left(x, y_{0}\right)}{\int_{x} f\left(x, y_{0}\right) d x}=\frac{h\left(x, y_{0}\right) / J\left(x, y_{0}\right)}{\int_{x} h\left(x, y_{0}\right) / J\left(x, y_{0}\right) d x}=\frac{h\left(x, y_{0}\right)}{J\left(x, y_{0}\right) k\left(y_{0}\right)}
$$

The density $f\left(x, y_{0}\right)$ describes the probability to obtain a pair $\left(x, y_{0}\right)$ i.e. the probability to obtain a $z$ lying on the surface $n(z)=y_{0}$. This is expressed by the relation

$$
f\left(x \mid y_{0}\right) d x=h\left(z \mid n(z)=y_{0}\right) d z
$$

If we substitute $(5.44)$ in $(5.41)$ we get

$$
\theta=\int_{x} p(\xi(z)) f\left(x \mid y_{0}\right) d x
$$

and we have transformed the integration over the $z$ space to an integration over the $x$ space. The estimation procedure is then to sample $m$ points $x_{i}$ from $f\left(x \mid y_{0}\right)$ and determining $z_{i}$ from the relation $z_{i}=\left(x_{i}, y_{0}\right)$. The estimate is then

$$
\theta=(1 / m) \Sigma p\left(z_{i}\right)
$$

Now we introduce an arbitrarily chosen density function $S(y)$, normal-

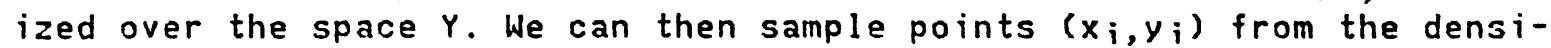
ty $f^{\prime}(x, y)=f\left(x, y_{0}\right) S(y)$ and since

$$
\int_{X \cdot Y} p\left(\xi\left[z\left(x, y_{0}\right)\right]\right) f^{\prime}\left(x, y_{0}\right) d x d y=\int_{X} p\left(\xi\left[z\left(x, y_{0}\right)\right]\right) d x \int_{Y} S(y) d y=\theta
$$

this change has not influenced the biasing. To overcome the difficulty of sampling from the conditional density function $f\left(x \mid y_{0}\right)$ we use the importance sampling trick. If the function $g(x, y)$ is a density function on $X \cdot Y$ then we have

$$
\theta=\int \frac{p\left(\xi\left[z\left(x, y_{0}\right)\right]\right) f^{\prime}(x, y)}{g(x, y)} g(x, y) d x d y
$$

If we choose $g(x, y)=f(x, y)=h(x, y) / J(x, y)$ the importance procedure is performed as follows: Sample m points $\left(x_{i}, y_{i}\right)$ from the unconditional density function $f(x, y)$. Solve the equations $\xi\left(z_{i}\right)=x_{i}$ and $n\left(z_{i}\right)=y_{0}$ to obtain $z_{i}$. Then the score is $p\left(z_{i}\right)$ and the weight $w\left(x_{i}, y_{i}\right)$. The estimate is

$$
\theta=(1 / m) \sum p\left(z_{i}\right) w\left(x_{i}, y_{i}\right)
$$

where $w\left(x_{i}, y_{i}\right)=\frac{f^{\prime}\left(x_{i}, y_{i}\right)}{g\left(x_{i}, y_{i}\right)}=\frac{h\left(x_{i}, y_{0}\right)}{h\left(x_{i}, y_{i}\right)} \cdot \frac{J\left(x_{i}, y_{i}\right)}{J\left(x_{i}, y_{0}\right)} \cdot \frac{s\left(y_{i}\right)}{k\left(y_{0}\right)}$

We have thus arrived at a sampling scheme which allows us to sample from the unconditional density function $f(x, y)$ and thus from $h(z)$. The 
function $S(y)$ gives us the possibility to reduce the variance and optimize the sampling scheme.

We give an example of the method by looking at the problem of determining the expected value of $p(z)$ when $z>0$ and $h(z)$ is a density function in $(-\infty, \infty)$. The condition $z>0$ can be defined as

$$
\begin{aligned}
y=n(z) & =1 & & \text { if } z>0 \\
& =-1 & & \text { if } z \leq 0
\end{aligned}
$$

The $Y$ space is then a two-element space and we choose $S(y)$ to be a binomial density with $\operatorname{Prob}(y=1)=\lambda$ where $\lambda$ may be chosen so that the variance is minimized. The transformation $\xi$ is chosen as

$$
x=\xi(z)=|z|
$$

Then the expectation may be written as

$$
\theta=\int_{z} p(z) h(z \mid n(z)=1) d z
$$

The Jacobian is $J(x, y)=1$ and $k\left(y_{0}\right)=\int h\left(x, y_{0}\right) d x={ }_{0} \iint_{0 h}(z) d z=p$. We then sample $z_{i}$ from $h(z)$ and determine $\left(x_{i}, y_{i}\right)$ from $(5.50)$ and $(5.51)$. If $z_{i}>0$ we get $x_{i}=z_{i}$ and $y_{i}=1=y_{0}$. The weight $w\left(x_{i}, y_{i}\right)$ is then (from (5.49)) $w\left(x_{i}, y_{i}\right)=\lambda / p$ and the score is $p\left(z_{i}\right)$. For $z_{i} \leq 0$ we get $x_{i}=-z_{i}, y_{i}=-1$ and score $p\left(-z_{i}\right)$ with the weight $w\left(x_{i}, y_{i}\right)=h\left(-z_{i}\right)(1-\lambda) /\left(p h\left(z_{i}\right)\right.$.

\subsection{FURTHER REFERENCES}

A general discussion of variance reducing techniques can be found in [26]. [4] and [13] are articles on orthonormal functions and conditional Monte Carlo. The use of Hermite expansions in Monte Carlo is explored in [9]. 
Chapter 6

APPLICATIONS

One of the main areas in which Monte Carlo calculations are used is in the field of particle transport. It is maybe not so surprisingly since it was in this field that the Monte carlo method first was used systematically. In this chapter we will look at some of the properties of the simulation of particle transport. First we give a formulation of the problem and then we will look at some typical sampling procedures and variance reduction techniques.

\subsection{THE PARTICLE TRANSPORT PROBLEM}

Particle transport simulations are concerned with the behaviour of particles e.g. neutrons, photons and electrons, when they interact with different materials. Some typical problems that arise are the shielding and the criticality problems. In the former we want to know how thick a shield should be so that we are protected from radiation. The latter problem is concerned with the behaviour of nuclear reactors.

The transport process can be described by an integro-differential equation in six dimensions known as the Boltzmann transport equation. This equation is solvable by analytical means only if we make a lot of approximations and most of the real problems are so complex that they have to solved with the Monte Carlo method. Usually, the Boltzmann equations is not solved directly with the Monte Carlo method. In fact, particle transport can be simulated without even referring to it. The Monte Carlo method used for solving the problem is most easily derived directly from the physical processes. This relies on the Markovian property of the transport i.e. the simulation of the physics at any point of a particle trajectory does not depend on how it came to that point. All that is required to simulate the history of the particle 3 is a probabilistic model of what may happen to the particle at each point in its history. This usually includes a description of the geometry, the material composition and a probabilistic model in terms of cross sections of the events that may happen. The physical simulation of particle transport is usually called an analog simulation or an analog Monte Carlo method.

3 With the history of a particle we mean a collection of events that describe the trajectory of the particle from its creation to its death. 
An analytical solution of the Boltzmann equation would give complete information of all the six variables time, energy, position and direction and other properties of interest could be derived from the solution. In a Monte Carlo simulation we do not achieve so many details but we rather get information about certain specified quantities such as energy deposition in various regions or the fluence in a certain interval of the phase space.

The treatment of each physical process in an analog simulation yields conceptually simple methods. However, it is not always an adequate way of looking at the problem. The analog computation can be slow and when we try to speed it up with various variance reducing techniques we often have to go back to the Boltzmann equation to justify the correctness of the new scoring schemes and to check that we have not introduced any bias in our estimators. These simulations that are not only based on the physical processes but use results derived from the Boltzmann equation are usually called weighted Monte Carlo methods since the difference between the two methods can be seen as appending a weight of importance to each particle.

\subsection{THE BOLTZMANN EQUATION}

In order to get a slight idea of what the Boltzmann equation looks like we state the time independent equation in its integral form. We let $p=(E, r, \Omega)$ denote a point in the phase space where $E$ is the kinetic ener$g y, r$ is the spatial coordinates and $\Omega$ is the direction of flight of the particle. Then the equation is

$$
F(p)=\int K\left(p^{\prime} \rightarrow p\right) F\left(p^{\prime}\right) d p^{\prime}+S(p)
$$

Here $F(p)$ is the collision density, $s(p)$ is the density distribution of the source particles and $K\left(p^{\prime} \rightarrow p\right)$ is the Boltzmann kernel. The kernel can be broken down into two kernels one transport kernel, $T$, and one collision kernel, $C$.

$$
K\left(p^{\prime} \rightarrow p\right)=T\left(r^{\prime} \rightarrow r \mid E^{\prime} \Omega^{\prime}\right) \quad C\left(E^{\prime} \Omega^{\prime} \rightarrow E \Omega \mid r\right)
$$

The transport kernel describes the probability for a particle emerging from $r^{\prime}$ with direction $\Omega^{\prime}$ and energy $E^{\prime}$ to make $i$ ts next collision at $r$ and the collision kernel is the probability that a particle entering a collision at $r$ with direction $\Omega^{\prime}$ and energy $E^{\prime}$ will emerge with an direction $\Omega$ and an energy $E$. 


\subsection{PARTICLE TRANSPORT}

The mechanism of simulating particle transport can be decomposed into a simulation of the transport and into the interactions of a single particle. The transport part can further be divided into a geometry part, a time part and a distance selecting part. To be able to estimate the quantities we are interested in we usually need some scoring of the events transport and interaction.

\subsubsection{Geometry}

The geometry of the problem has to be described. Boundaries of regions with different materials and boundaries of regions used for the scoring are specified with one or more quadratic surfaces (or sometimes with toroids). These surfaces are either treated as a general quadratic surface or as the simpler surfaces such as planes, cylinders or spheres. The regions can be specified by their boundary surfaces and by an orientation to each of these surfaces.

The particle flight is usually best described in Cartesian coordinates even though many problems have cylindrical or spherical symmetries. The direction of $f l i g h t$ is given by a unit vector $(u, v, w)$. The transport of the particle a distance $t$ from the initial point $(x, y, z)$ to the new position $\left(x^{\prime}, y^{\prime}, z^{\prime}\right)$ is then simply

$$
x^{\prime}=x+t u ; \quad y^{\prime}=y+t v ; \quad z^{\prime}=z+t w ;
$$

In Cartesian coordinates the direction vector do not change along flight paths (if the particle is uncharged). However, it does change at scattering events. The flight distance $t$ depends on several physical properties. One of these is the material in which the particle is. Thus, before we can transport the particle we have to check with the given geometry so that the particle trajectory do not cross any boundary. Therefore, since the geometry routines are called many times they should be fast and accurate.

\subsubsection{Iime}

Time dependence is easily included in a Monte Carlo calculation. After each flight the time is advanced and the scoring is then made in the appropriate time bins. 


\subsubsection{Next interaction}

The transport distance between interactions is governed by the mean free path, $\lambda$, of the particle. The $\lambda$ is given in terms of a macroscopic total cross section, $\Sigma_{t}$, according to

$$
\lambda=1 / \Sigma_{t}
$$

The mean free path is in general a function of the material and the energy.

The probability that a particle starting at $x_{0}=0$ will suffer its next collision in some distance $\Delta x$ about $x$ is given by

$$
p(x) \Delta x=(\Delta x / \lambda(x)) \operatorname{Exp}\left(-0 \int^{x} d x^{\prime} / \lambda\left(x^{\prime}\right)\right)
$$

The distribution function is then

$$
P(x)=1-\operatorname{Exp}\left(-0 \int x d x^{\prime} / \lambda\left(x^{\prime}\right)\right)
$$

If we use the direct sampling method we get

$$
0 \int x^{\prime} / \lambda\left(x^{\prime}\right)=-\ln \xi
$$

where $\xi$ is uniformly distributed in $[0,1]$.

From this relation we may locate the next interaction point $x$. In the case when we are transporting particles that have a constant cross section between interactions this procedure is simple. We just calculate the next interaction point as $x=-\ln \xi / \Sigma_{t}$ and if this distance is greater than the distance to the point where the cross section changes ( e.g. a boundary) we transport the particle to that point and repeat the procedure with the new cross section. If the cross section changes along the path of the particle we can, in the case where the cross section decreases with the energy (and hence with the distance), use the following trick to sample the next interaction point. We introduce a fictious event with a cross section $\Sigma_{t}, f i c t(x)$. This event is a straight ahead scattering i.e. no interaction at all. The magnitude of $\Sigma_{t, f i c t}$ is constant along the path between $x_{0}$ and $x$ and is given by (see fig 3 )

$$
\Sigma_{t, f i c t}(x)=\Sigma_{t, \text { real }}(x)+\Sigma_{f i c t}(x)=\Sigma_{t, \text { real }}\left(x_{0}\right)
$$

We sample the next "interaction" point from (6.7) using $\Sigma_{t, f i c t}$ instead of $\Sigma_{t}$. When this interaction point is reached we generate a uniform deviate $\xi$ and if the relation

$$
\xi>\Sigma_{t, \text { real }}(x) / \Sigma_{t, \text { real }}\left(x_{0}\right)=1-\Sigma_{f i c t}(x) / \Sigma_{t, \text { real }}\left(x_{0}\right)
$$

is true we say that the interaction was fictious otherwise it was real. 


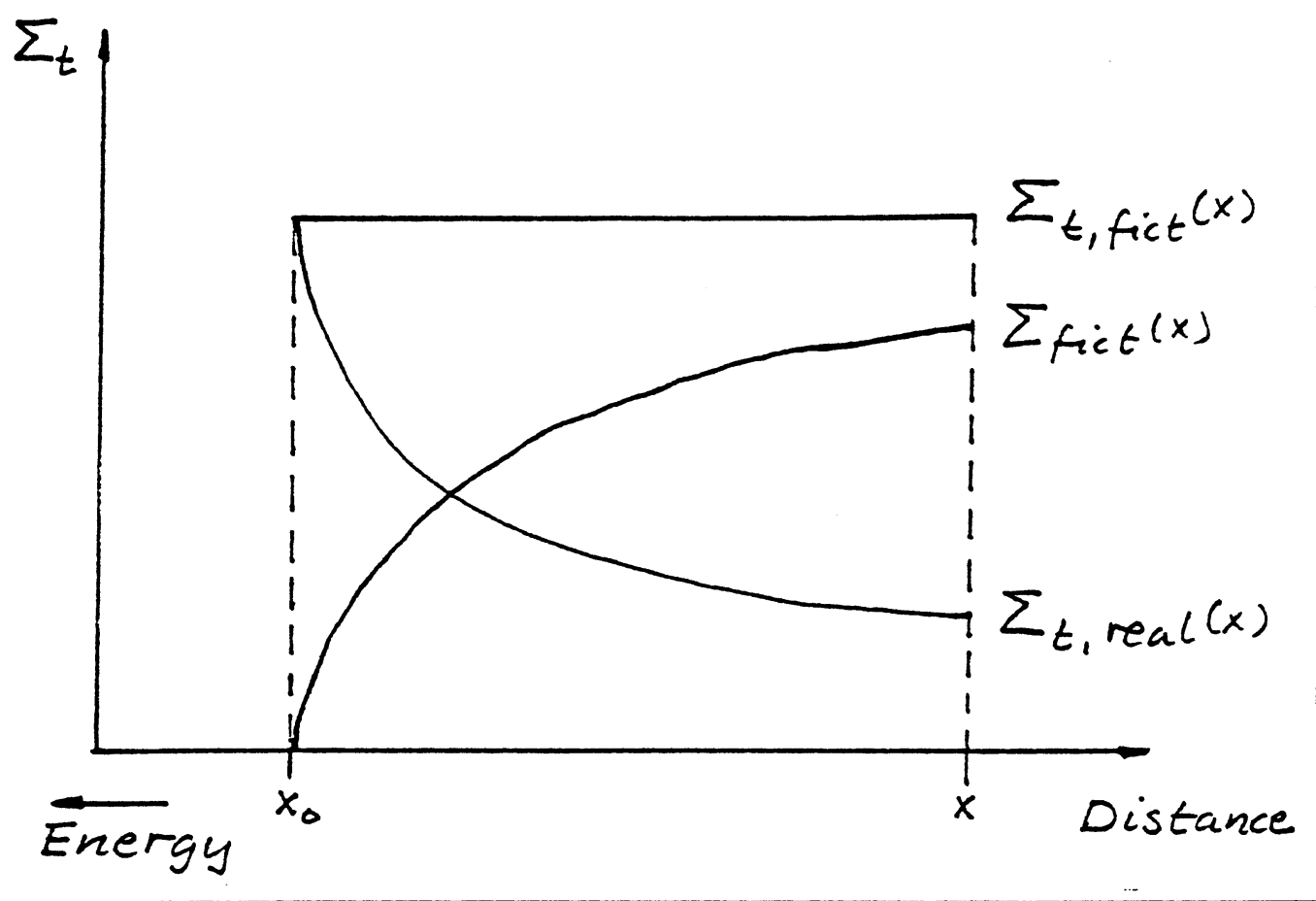

Figure 3: Fictious interactions

\subsubsection{Interaction}

When a point of interaction is reached it must be decided which of the possible interaction processes that has occurred. The probability that a given type of interaction has occurred is proportional to its cross section $\sigma$. If there are $n$ possible interactions then the probability that interaction $i$ occur is

$$
\text { Prob( Interaction type i) }=\sigma_{i} / \sigma_{t}
$$

where $\sigma_{t}=\Sigma \sigma_{i} \quad i=1, \ldots, n$.

The interaction type is then selected by generating a uniform deviate $\xi$ and the type is $k$ where $k$ satisfy the relation

$$
\sum_{i=1}^{k-1} \sigma_{i} \leq \xi \sigma_{t}<\sum_{i=1}^{k} \sigma_{i}
$$

After the type is chosen we calculate or sample from other distributions the properties of the particles that are emitted. And then we are back to the transport phase again. 
One interaction is worth some comments. When we are dealing with neutrons there is a probability that the neutron is captured. This could be handled in the obvious way just to let the history of the particle end with the capture. But this could lead to a great loss of particle tracks and thus getting lower accuracy. One way to avoid this is to exclude the possibility of capture in such a way that we still account for it. This is done by reducing the weight of the particle with the non-capture probability $\left(\sigma_{t}-\sigma_{c}\right) / \sigma_{t}\left(\sigma_{c}\right.$ is the cross section for capture) after a collision. The interaction is then sampled from the other possible types with probabilities normalized to exclude capture.

\subsubsection{Estimators}

The mostly used estimators in Monte Carlo transport codes are of four types. These are the collision estimator, the last-event estimator, the track-length estimator and the next-event estimator or the point detector. We demonstrate the last two of them by looking at the estimation of the particle fluence as a function of $x$ position and energy. In the following we let $M$ denotes the total number of source particles and $w t_{i}$ is the weight of the $i$ th particle. The track-length estimator calculate the flux density in a given arbitrary volume in space at a location of interest. The estimator is

$$
\phi(E, r)=\sum_{i=1}^{N} 1_{i} w t_{i}, \Delta v \Delta E M
$$

where $\Delta v$ is the volume element, $\Delta E$ is an energy bin

$l_{i}$ is the track-length of the $i$ th particle and

$N$ is the number of particles in $\Delta v$ with energies in $\Delta E$ about $E$.

This is often a very good estimator especially when the collision rate is low in the volume elements of interest. The next-event estimator is often better than the track-length estimator and it is capable of determining the flux density at a point in space. However, its use often result in an increase of the computing time. The estimator is composed of two contributors: one from the uncollided response and one from the collided. The two terms can be written as

$$
\begin{gathered}
\phi(E, r)_{u c}=\sum_{i=1}^{N} \operatorname{Exp}\left(-\Sigma_{t}(E)\left|r_{i}-r\right|\right) w t_{i} /\left(4 \pi\left|r_{i}-r\right|^{2} M\right) \\
\phi(E, r)_{c}=\sum{\underset{i=1}{g}\left(E^{\prime} \rightarrow E, \Omega^{\prime} \rightarrow \Omega\right) \operatorname{Exp}\left(-\Sigma_{t}(E)\left|r_{i}-r\right|\right) w t_{i} /\left(\left|r_{i}-r\right|^{2} M\right)}^{N}
\end{gathered}
$$

where $r_{i}$ is the position of the source or the produced particles $r$ is the position of the detector 
$N$ is the the total number of source and produce particles in the energy interval $[E, E+\Delta E]$ and the total number of particle collisions respectively.

$g\left(E^{\prime} \rightarrow E, \Omega^{\prime} \rightarrow \Omega\right)$ is the probability that an incident particle of energy $E^{\prime}$ and direction $\Omega^{\prime}$ will emerge from the interaction with energy $E$ and direction $\Omega$ towards the detector.

\subsection{VARIANCE REDUCTION}

Apart from the common variance reduction techniques discussed in the previous chapter there are some special techniques which are often used in transport simulations. These methods mainly try to assure that we get enough contribution to the estimators in the regions that are the most important. This involves a control of the number of particle tracks. We do not want to have too few because then we get bad statistics but on the other hand we do not want to have too many since then we probably spend a lot of time on particles that contribute little to the result.

\subsubsection{Splitting and Russian roulette}

The basis for this technique is that when particles migrate in an important direction they are increased in number to provide better sampling and when they are moving in the opposite direction they are killed in an unbiased manner. To increase the number of particles we split one particle into $v$ new identical particles. If the split particle has weight wt then the new particles must emerge from the splitting event with the weights $w t / y$. We then preserve the weight and thus do not introduce any bias. We can choose the number of new particles to be an integer number but we can also split into fraction of particles. If $y$ is not an integer and $n<v<n+1$ then we split into $n+1$ new particles with probability $\nu-n$ and into $n$ new ones with probability $n+1-v$.

If we want to decrease the number of particles we use a method called Russian roulette. The particles are killed with a probability $1-v^{-1}$ or survive with probability $v^{-1}$. If the particle survive we increase its weight with a factor $v$. The number $v$ is preferably the same $v$ as used in the splitting.

This is a reliable variance reduction method, at least if these two techniques are used together, and consequently it is also widely used. If we let the distance between the splitting events tend to zero then this method will be a special case of the next method. 


\subsubsection{The exponential transform}

The exponential transform increases the particle histories in the desired direction by a "path-length stretching". This is achieved by artificially increase the distance between collisions in the desired direction while at the same time decrease this distance in the opposite direction. The path length is changed by artificially reduce the total macroscopic cross section $\Sigma_{t}$. If we let $\mu$ denote the cosine of the angle between the preferred direction and the line of flight of the particle and $\alpha$ is a parameter then the fictious cross section $\Sigma_{e x}$ is

$$
\Sigma_{\text {ex }}=\Sigma_{t}(1-\alpha \mu)
$$

The distance to the next interaction is then sampled from (6.T) with $\Sigma_{e x}$ instead of $\Sigma_{t}$. In order to not introduce any bias we then have to adjust the weight of the particle. From the probabilities for collision or no collision in $d x$ about $x$ we derive the following two conservation relations from which we determine the necessary weights $w_{c}$ and wuc

$$
\Sigma_{t} \operatorname{Exp}\left(-\Sigma_{t} x\right) d x=w_{c} \Sigma_{\operatorname{ex}} \operatorname{Exp}\left(-\Sigma_{e x} x\right) d x
$$

and

$$
\operatorname{Exp}\left(-\Sigma_{t x}\right) d x=w_{u c} \operatorname{Exp}\left(-\Sigma_{\text {ex }} x\right) d x
$$

The weights in the both cases are then

$$
\begin{gathered}
w_{c}=\left(\operatorname{Exp}\left(-\Sigma_{t} \alpha \mu x\right) /(1-\alpha \mu)\right. \\
w_{\text {uc. }}=\operatorname{Exp}\left(-\Sigma_{t} \alpha \mu x\right)
\end{gathered}
$$

The exponential transform should be used with care since an improperly use may result in strongly biased results.

\subsubsection{Particle cutoffs}

When a particle reaches a point where it does not make any significant contribution to any estimator it is better to discard the particle instead of wasting computing time on following it further. And anyhow the histories must be terminated in one way or another. Thus, we must have some mechanism for killing particles. Russian roulette has already been discussed as a killing method. Another common way to kill particles is to use zero-importance regions. As soon as we reach a such region the particle is discarded. One common such zero-importance region is the outside-world i.e. the complement to the geometry we are interested in. A third mechanism for killing is to have cutoff values for energy, time and/or weight. When the values of a certain particle passes these cutoff values its history is simply terminated. These cutoff techniques are of ten used with a good result but one have to be careful. In the case of weight cutoffs the setting of the cutoff levels are difficult to handle and the final setting is often a result of trial-and error. 


\subsection{SOFTWARE}

There is a wide range of Monte Carlo programs for particle transport. Most of them have been produced to be used as research tools in connection with particle accelerators and nuclear industry. The codes can be classified after which particles they can transport and after the energy limits that the physical models are relevant for. We give here some examples of codes characterized after which particles that are transported.

MORSE[14] (Oak Ridge National Laboratory) is a neutron-photon transport code. It can handle particles with energies up to $20 \mathrm{MeV}$. MORSE is a weighted Monte Carlo code. The geometry is described with a generalized three dimensional package and time dependence can be handled. MORSE can e.g. be used to solve problems such as fusion reactor shielding or high energy nuclear instrumentation design.

An example of an analog Monte Carlo program is the EGS Code system[17] (SLAC/CERN, Geneva). EGS stands for electron-gamma shower and it simulates the cascade produced by photons and electrons-positrons. EGS can in principle handle source particles of any energy it depends only on the capability of creating appropriate material data sets for the energy levels of interest. The geometry in EGS is, at present, describable with the four surfaces planes, cylinders, spheres and cones. Some examples of the use of EGS is the calculation of the dose distribution in the human body and calculations on synchrotron radiation.

KASPRO[39] (CERN, Geneva) is an example of a code that treats hadronic cascades. Particles in the energy range $50 \mathrm{MeV}$ to $1 \mathrm{TeV}$ are transported and the geometry is described with cylinders and planes. KASPRO is a weighted Monte carlo program and it could for example be used to calculate the particle production in extended targets and radiation doses due to the hadronic cascade.

\subsection{FURTHER REFERENCES}

[7] and [45] are general text books on particle transport problems. A pioneer work in the application of Monte Carlo techniques to physics can be found in [42]. The connection between health physics and particle transport is treated in [37]. In [46] Spanier examines various estimators for transport problems. 
Appendix A

PROBABILITY THEORY

\section{A. 1 PROPERTIES OF RANDOM VARIABLES}

This appendix will give a short repetition of the probability theory which is the basis for the Monte carlo calculations and for the justification of the results. For those who have forgotten their elementary probability theory it might be worthwhile reading an introductory book in the subject such as [5] or [10].

A random variable, $x$, is a function from a sample space $\Omega$ to the real numbers.

$$
x: \omega \rightarrow \mathbb{R}, \omega \in \Omega
$$

We can also have random variables which are functions of random variables e.g. $y=f(x)=\sqrt{x}$.

A random variable has a distribution function, $F$, which is defined as

$$
F(x)=\operatorname{Prob}(x \leq x)=\int_{S} d F(t)
$$

where $s=\Omega \cap\{t \mid t \leq x\}$

A density function, $f$, is a function defined on $x$ and satisfying the two conditions

i) $\forall u: f(u) \geq 0$

ii) $\int_{x} d F(u)=1$

where we have used that $f(u) d u=d F(u)$

We also have the relation

$$
\operatorname{Prob}(x=x)=f(x)
$$

In the case when we have functions of several random variables we define the joint distribution function as 


$$
F(x, y)=\operatorname{Prob}(x \leq x, y \leq y)=\iint_{S} d F(x, y)=\int_{-\infty}^{x} \int_{-\infty} y f(u, v) d u d v
$$

and an analog definition for more than two variables.

In the case of multivariate distributions we are often interested in the marqinal distribution of some variable. The marginal distribution in the bivariate case is

$$
F_{x}(x)=\operatorname{Prob}(X \leq x, y \leq \infty)=\iint_{S} d F_{x}(x, y)=\int_{-\infty}^{x} \int_{-\infty}^{\infty} f(u, v) d u d v \quad \text { (A. 6) }
$$

Another concept of interest is the conditional probability i.e. the probability for the random variable $X$ (say) when we know the value of the random variable $Y$ (say). The relation between joint, marginal and conditional density functions is

$$
p(x, y)=p_{y}(y) p(x \mid y)
$$

If the conditional $\operatorname{Prob}(x \mid y)=\operatorname{Prob}(x)$ joint distribution functions :

probability of $X$ given $Y$ does not depend on $Y$ i.e. then we say that $X$ and $Y$ are independent. Thus the will just be the product of the different density

$$
p\left(x_{1}, x_{2}, \ldots\right)=p\left(x_{1}\right) p\left(x_{2}\right)
$$

The expectation, $E$, of a random variable, $x$, is defined as

$$
E[x]=\int_{x} x d F(x)
$$

or if we have function of a random variable the expectation is

$$
E[g(x)]=\int_{x} g(x) d F_{x}(x)
$$

The next higher moment is used to define the variance, Var, of a random variable. It is defined as

$$
\operatorname{Var}[X]=E\left[(X-E[X])^{2}\right]=E\left[X^{2}\right]-(E[X])^{2}
$$

The positive square root of the variance is called the standard deviation.

The covariance, Cov, of two random variables, $X$ and $Y$, is

$$
\operatorname{Cov}[X, Y]=E[(X-E[X])(Y-E[Y])]=E[X Y]-E[X] E[Y]
$$

If $X$ and $Y$ are independent random variables then $\operatorname{cov}[X, Y]=0$ 


\section{A.2 COMMON DISTRIBUTIONS}

As an example of the definitions above we look at some often encountered distributions.

1. Uniform distribution.

$x$ is uniformly distributed over $[a, b]$ and has the density function

$$
f(x)=1 /(b-a)
$$

and the distribution function

$$
F(x)={ }_{a} \int x f(t) d t=(x-a) /(b-a)
$$

The mean and the variance of $x$ is

$$
E[x]=(a+b) / 2 \quad \operatorname{Var}[x]=(b-a) / 12
$$

2. Binomial distribution.

Let the probability of choosing one of two objects be $p$ (e.g. Prob ( a toss of a coingives face) $=p$ ). If $x$ is the number of times that this object has been chosen in $n$ trials then

$$
\operatorname{Prob}(x=j)=p(j)=(\hat{j}) p^{j}(1-p)^{n-j} \quad j=0,1, \ldots, n
$$

This is a binomial distribution and the density function is $p(x)$. The distribution function is then the sum

$$
P(i)=\sum_{j=1} p(j)
$$

The mean and the variance is

$$
E[x]=n p \quad \operatorname{Var}[x]=n p(1-p)
$$

3. Exponential distribution.

Let the random variable $x \in[0, \infty[$ and let the parameter $\lambda>0$. Then we say that $x$ is exponential distributed if it has the density function

$$
f(x)=\lambda \operatorname{Exp}(-\lambda x)
$$

The distribution function is then

$$
F(x)=1-\operatorname{Exp}(-\lambda x)
$$

The mean and the variance is easy calculable and we get

$$
E[x]=1 / \lambda \quad \operatorname{Var}[x]=1 / \lambda^{2}
$$

4. Normal distribution.

The normal distribution with parameters $\mu$ and $\sigma$ is an important distribution. For $x \in]-\infty, \infty[$ the density function is

$$
f(x)=(1 /(\sigma \sqrt{2 \pi})) \operatorname{Exp}\left(-(x-\mu)^{2} /\left(2 \sigma^{2}\right)\right)
$$

and the distribution function is 


$$
F(x)=-\infty \int x f(t) d t
$$

The mean and the variance are

$$
E[x]=\mu \quad \operatorname{Var}[x]=\sigma^{2}
$$

If $\mu=0$ and $\sigma=1$ then we call the normal distribution for the standardized normal distribution.

\section{A. 3 LIMIT THEOREMS}

When we want to assure the correctness of the results in a Monte Carlo calculation we use two main theorems from the probability theory namely the Central Limit Theorem, CLT, and the Law of Large Numbers, LLM.

The Central limit theorem states that if we have a sequence $\left\{x_{i}, 1 \leq i \leq n\right\}$ of independent and identically distributed, id, random variables and with

1. $a<b$

$$
\text { 2. } s_{n}=\sum_{i=1}^{n} x_{i}
$$

then

$$
\lim _{n \rightarrow \infty} \operatorname{Prob}\left(a<\left(S_{n}-n m\right) /(\sigma \sqrt{n}) \leq b\right)=(1 / \sqrt{2 \pi})_{a} \int^{b} \operatorname{Exp}\left(-x^{2} / 2\right) d x
$$

where $E\left[S_{n}\right]=n m$ and $\operatorname{Var}\left[S_{n}\right]=n \sigma^{2}$

Thus the sum of $n$ independent and identically distributed random variables has a normal distribution with mean $\mathrm{nm}$ and variance $n \sigma^{2}$

The Law of Large Numbers is closely related to the central limit theorem and it states that under the same conditions as in (A. 13) and for an arbitrary $\in>0$

$$
\lim _{n \rightarrow \infty} \operatorname{Prob}\left(\left|\left(s_{n} / n\right)-m\right|<\epsilon\right)=1
$$


Appendix B

MATHEMATICAL BASIS

\section{B. 1 MATHEMATICAL PROPERTIES}

The theory of the Monte carlo method is based on the fact that we can produce truly random numbers. As we discussed in chapter 2 true random numbers are difficult to generate and we almost always use pseudo-random numbers instead. But these generators produce a determined sequence of numbers and hence the results of the computations are also determined. Statistical argument about deterministic numbers are quite irrelevant so if we use pseudo-random numbers we can not say anything about e.g. convergence rate or variance. The Monte Carlo method thus seems to be unusable. But we can overcome this difficulty in two ways. The first way is just to use the theory as it stands since even if we use pseudo-random numbers the computations show that we get the same convergence rate and the same accuracy as if we had used true random numbers. This is due to the fact that pseudo-random numbers usually have the properties that we need to impose on the sequence in order to be able to prove more rigorous error bounds. The second approach is the quasi-random number approach. Instead of using pseudo-random numbers we use sequences which do not pretend to imitate the randoinness but has a well defined and a suitable distribution (e.g. equidistribution modulo 1). For these numbers we can then make statements about accuracy and convergence of computations. We give here the main results in the quasi-Monte Carlo theory $[50,51]$.

We look at the problem of evaluating a finite dimensional integral. We assume that the domain of integration has measure 1 and that we have a sequence $\Xi=\left\{\xi_{i}\right\} \quad i=1, \ldots, m$ of equidistributed points in that domain. We choose the hyper-unit cube $u^{k}=\left\{x \mid 0 \leq x_{i}<1\right.$ for $\left.i=1, \ldots, k\right\}$ as the domain.

The imperfection of the equidistribution of the sequence $\Xi$ is described by the local discrepancy. Let $v_{m}(z)$ be the number of points in $\Xi$ that 1 ie in the hyperbrick $\left\{\xi \mid 0 \leq \xi ;<a_{i} \leq 1\right.$, for $\left.i=1, \ldots, k\right\}$. Then the local discrepancy of the sequence $\Xi$ at $z$ is defined as

$$
\Delta_{m}(z)=v_{m}(z) / m-a_{1} a_{2} \cdots a_{k}
$$

From the local discrepancy we can derive different measures of the imperfection. The extreme discrepancy, $D_{m}(\equiv)$, is

$$
D_{m}(\equiv)=\sup _{z \in U^{k}}\left|\Delta_{m}(z)\right|
$$


and the mean-square (or $\left.L^{2}\right)$ discrepancy, $I_{m}(\Xi)$, is given by

$$
T_{m}(\Xi)=\left(\int_{U k}\left|\Delta_{m}(z)\right|^{2} d z\right)^{1 / 2}
$$

With these measures we can obtain upper bounds for the error in the approximation of the integral of $f(z)$ over $u^{k}$. We call this error for $\delta_{m}(f)$ and it is derived as

$$
\delta_{m}(f)=\left|\int_{U^{k}} f(z) d z-\sum_{m k=0}^{m-1} f\left(z_{k}\right)\right|
$$

To be able to express the error bound we also need the notation of variation. The k-dimensional total variation of a function $f$ in $u^{k}$, $V_{k}(f)$, is defined by considering $k$ arbitrary partitions, $p_{i}$, of $U$ at the points $0=x_{i o}<x_{i 1}<\ldots<x_{i n}(i)=1 \quad(i=1, \ldots, k)$. Then we define

$$
\begin{aligned}
\left.\delta_{z(}\right) \phi(z)= & \phi\left(z_{1}, \ldots, x_{i j}(i), \ldots, z_{k}\right)- \\
& -\phi\left(z_{1}, \ldots, x_{i}(j(i)-1), \ldots, z_{k}\right)
\end{aligned}
$$

and $\delta_{z}(1), z(2), \ldots, z(k) \phi(z)=\delta_{z(1)} \cdots \delta_{z(k)} \phi(z)$.

The total variation $V_{k}(f)$ is then

$$
v_{k}(f)=\sup _{j(1)=i} \sum_{j(k)=1}^{n(k)}\left|\delta_{z}(1) \ldots z(k) g(z)\right|
$$

where the supremum is taken over all partitions $p_{i}$. If $V_{k}(f)$ is finite we say that $f$ has a bounded variation.

To clarify the notation we look at the two-dimensional case with the partitions $0=x_{1}<\ldots<x_{m}=1$ and $0=y_{1}<\ldots<y_{n}=1$. We then get $v_{2}(f)$ as

$$
V_{2}(f)=\sum_{i=1}^{m} \sum_{i=1}^{n}\left|f\left(x_{i}, y_{i}\right)-f\left(x_{i}-1, y_{i}\right)-f\left(x_{i}, y_{i}-1\right)+f\left(x_{i}-1, y_{i}-1\right)\right|
$$

This definition of variation could give quite strange results. In two dimensions, for example, we see that for a function which does not depend on $y$ (say) the variation is zero no matter how much the function varies with $x$. To overcome this deficiency we make a slight modification to the definition above. Let the function $g$ be the $h$-dimensional function obtained by $f i x i n g$ any $(k-h)$ of the $z_{i}$ 's to 1 in the $k$-dimensional function $f$. Then we say that the function $f$ is of bounded variation if $V_{k}(f)$ is finite and if $V_{h}(g)$ is also finite for all h<k. Further, we define the selecting function $s(h)$ to be any choice of $h$ of the coordinates $z_{i}$. Then the sequence $\Xi_{s}(h)$ is the sequence $\Xi$ but with $h$-dimensional points with the coordinates selected by $s(h)$.

We are now ready to give the error bounds for $\delta_{m}(f)$ in terms of the discrepancy and the variation. If the function $f$ is of bounded variation 
(in the latter sense) then the error in the integration of $f$ by a quasi-Monte carlo method is bounded by

$$
\delta_{m}(f) \leq \sum_{h=1}^{k} \sum_{s(h)} D_{m}\left(\Sigma_{s}^{\prime}(h)\right) V_{h}\left(f_{s}(h)\right)
$$

where $f_{s}(h)=g$ as defined above.

or expressed with the mean-square discrepancy

$$
\delta_{m}(f) \leq \sum_{h=1}^{k} \sum_{s(h)} T_{m}\left(\Xi_{s}^{\prime}(h)\right) W_{h}\left(f_{s}(h)\right)
$$

where

$$
W_{h}\left(f_{s}(h)\right)=\left(\int_{U^{k}}\left|\partial / \partial z_{i}\left(f_{s(h)}\right)\right|^{2} d z\right) 1 / 2
$$

the differentiation being performed once for each $z$ i selected by $s(h)$.

These results could justify the use of pseudo-random numbers or quasi-random numbers when the sequence of points used in the calculations has been tested for its discrepancy. So if we want to perform a Monte Carlo calculation with pseudo-random numbers we should not make any test for randomness of the sequence but examine its discrepancy.

\section{ACKNOWLEDGEMENTS}

This report is the author's thesis for his Masters degree in Electrical Engineering and Applied Physics. His studies has been performed at the Technical Highschool in Linkoping, Sweden and particular for this present work at the Radioprotection Group of the Health and Safety Division, CERN, Geneva, Switzerland.

I wish to thank the HS division and especially Graham stevenson in the HS/RP group for making it possible for me to accomplish this work at CERN as a Technical Student. I also wish to thank Ake Bjorck, University of Linkoping for his participation in foregoing discussions and in the examination. 


\section{REFERENCES}

1. Ahrens,J.H. and Dieter,U.: Extensions of Forsythe's Method for Random Sampling from the Normal Distributions, Math.Comp., 27, pp 927-937, (1973).

2. Ahrens, J.H. and Dieter,U.: Computer Methods for Sampling from the Exponential and Normal Distributions, CACM, 15, pp 873-882, (1972).

3. Ahrens, J.H., Dieter,U. and Grube,A.: Pseudo-Random Numbers: A New Proposal for the Choice of Multiplicators, Computing, 6, pp 121-138, (1970).

4. Bogues, K., Morrow,C.R. and Patterson,T.N.L.: An Implementation of the Method of Ermakov and Zolotuk in for Multiple Integration and Interpolation, Numer. Math., 37, pp 49-60, (1981).

5. Brownlee,K.A.: Statistical Theory and Methodology, John Wiley, New York, 2nd ed.

6. Buffon : Essai d'arithmetique morale, 1777.

7. Carter,L.L. and Cashwell,E.D.: Particle Transport Simulation with the Monte Carlo Method, ERDA critical review series, TID-26607, 1975.

8. Chen,M-S. and Peierls,R.F.: A Mapping Technique for Efficient Random Event Generation with Constraints, J.Comp.Phys., 16, pp 195-220, (1974).

9. Chorin,A.J.: Hermite Expansions in Monte-Carlo Computations, J.Comp.Phys., 8, pp 472-482, (1971).

10. Chung,K.L.: Elementary Probability Theory with Stochastic Processes. Springer-Verlag, New York, 1974.

11. Dieter,U.: Pseudo-Random Numbers: The Exact Distribution of Pairs, Math.Comp., 25, pp 855-883, (1971).

12. Dieter, U. and Ahrens, J.H.: An Exact Determination of Serial Correlation of Pseudo-Random Numbers, Numer. Math., 17, pp 101-123, $(1971)$.

13. Dubi,A. and Horowitz,Y.S.: The Interpretation of Conditional Monte Carlo as a form of Importance Sampling, SIAM J.Appl.Math., 36, pp $115-122$, (1979). 
14. Emmet,M.B.: The MORSE Monte Carlo Radiation Transport System, ReP. ORNL-4972, 1975.

15. Everett,C.J. and Cashwel1,E.D.: A Monte Carlo Sampler, Rep. LA-5061-MS, Los Alamos scientific laboratory, Los Alamos, New Mexico, 1972 .

16. Everett,C.J. and Cashwell,E.D.: A Second Monte Carlo Sampler, Rep. LA-5723-MS, Los Alamos scientific laboratory, Los Alamos, New Mexico, 1974 .

17. Ford,R.L. and Nelson,W.R.: The EGS Code System, Rep. SLAC-210, 1978.

18. Forsythe, G.E.: Von Neumann's Comparison Method for Random Sampling from the Normal and Other Distributions, Math.Comp., 26, pp $817-826,(1972)$.

19. Franklin,J.N.: Deterministic Simulation of Random Processes, Math.Comp., 17, pp 28-59, (1963).

20. Freeze,R.A.: An Analysis of Baseball Batting order by Monte Carlo Simulation, Oper.Res., 22, pp 728-735, (1974).

21. Freiberger,W. and Grenander,U.: A Short Course in Computational Probability and Statistics, Springer-Verlag, New York, 1971.

22. Friedman, J.H. and Wright,M.H.: A Nested Partitioning Procedure for Numerical Multiple Integration, ACM Trans.Math.Soft., 7, pp 76-92, $(1981)$.

23. Haber, S.: Numerical Evaluation of Multiple Integrals, SIAM Review, 12, pp 481-526, (1970).

24. Halton,J.H.: A Retrospective and Prospective Survey of the Monte Carlo Method, SIAM review, 12, pp 1-63, (1970).

25. Halton, J.H.: On the Efficiency of certain Quasi-Random Sequences of Points in Evaluating Multidimensional Integrals, Numer.Math., 2, pp 84-90, (1960).

26. Hammersley,J.M. and Handscomb,D.C.: Monte Carlo Methods, Me thuen, London, 1964.

27. James.F.: Monte Carlo Theory and Practice, Rep. CERN/O0/80/6 CERN data handling division, Geneva, 1980.

28. Jansson, B.: Random Number Generators, Almquist och Wiksel1, stockholm, 1966.

29. Kinderman, A.J. and Monahan, J.F.: Computer Generation of Random Variables Using the Ratio of Uniform Deviates, ACM Trans.Math.Soft., 3, pp 257-260, (1977). 
30. Knuth, D.E.: The Art of Computer Programmina, Vol 2, Seminumerical Algorithms, Addison-Wesley, Reading, Massachusetts, 1969.

31. Knuth,D.E.: Construction of a Random Sequence, BIT, 4, pp 246-250, (1965).

32. Lehmer, D.H.: Mathematical Methods in Larqe-Scale Computing Units, Proc.Second Symposium on Large-Scale Digital Calculating Machinery, Harvard University Press, Cambridge, Massachusetts, pp 141-146, 1951.

33. Marsaglia,G.: Random Numbers Fall Mainly in the Planes, Proc.Nat.Acad.Sci., 61, pp 25-28, (1968).

34. Metropolis, N. and Ulam, S.M.: The Monte Carlo Method, J.Amer.Statis.Assoc., 44, pp 335-341, (1949).

35. Meyer,H.A.: Symposium on Monte Carlo Methods, John Wiley, New York, 1956.

36. Monahan,J.F.: Extensions of von Neumann's Method for Generating Random Variables, Math.Comp., 33, pp 1065-1069, (1979).

37. Nelson,W.R. and Jenkins,T.M., ed.: Computer Techniques in Radiation Transport and Dosimetry, Plenum Press, New York, 1980.

38. Page,E.S.: on Monte Carlo Methods in Congestion Problems: I. Searching for an optimum in Discrete Simulations, Oper.Res., 13, pp 291-299, (1965).

39. Ranft,J.: The Hadronic Cascade Code KASFRO, Rep. CERN Lab IIRA/75-1, 1975.

40. Robertson, I. and Walls, L.A.: Random Number Generators for the Normal and Gamma Distributions using the Ratio-of-Uniform Deviates Method, Rep. AERE-R 10032, (1981).

41. Roth,K.F.: On Irreqularities of Distributions, Mathematika, 1, pp 73-79, (1954).

42. Rudstam, G.: Spallation of Medium Weight Elements, Thes is, Uppsala Univ., 1956.

43. Shreider,Y.A., ed.: The Monte Carlo Method, Pergamon Press, Oxford, 1966.

44. Sobol', I.M.: The Monte Carlo Method, University of Chicago Press, Chicago, 1974.

45. Spanier,J. and Gelbard,E.M.: Monte Carlo Principles and Neutron Iransport Problems, Addison-Wesley, Reading, Massachusetts, 1969.

46. Spanier,J.: Iwo Pairs of Families of Estimators for Transport Problems, J.SIAM Appl.Math., 14, pp 702-713, (1966). 
47. Stroud,A.H.: Approximate Calculation of Multiple Integrals, Prentice-Hall, New Jersey, 1971.

48. "Student": Probable Error of a Correlation Coefficient, Biometrika, 6, pp 302-310, (1908).

49. Weyl,H.: Uber die Gleichverteilung von Zahlen mod. Eins, Math.Ann., 77, pp 313-352, (1916).

50. Zaremba,S.K.: The Mathematical Basis of the Monte Carlo Method and Quasi-Monte Carlo Methods, SIAM review, 10, pp 303-314, (1968).

51. Zaremba,S.K., ed.: Applications of Number Theory to Numerical Analysis, Proceedings of the Symposium at the Centre for Research in Mathematics, University of Montreal; Academic Press, New York, 1972. 